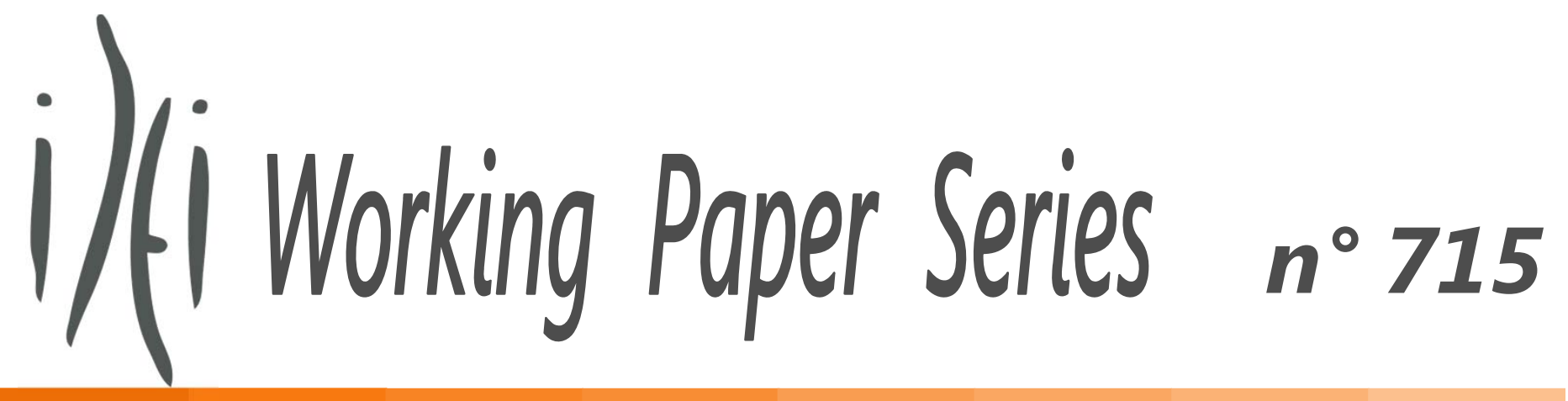

May, 2012

\title{
" Stability and Fairness in Models with a Multiple Membership"
}

\author{
Michel Le Breton, \\ Juan D. Moreno-Ternero, \\ Alexei Savvateev \\ and
}

Shlomo Weber 


\title{
Stability and Fairness in Models with a Multiple Membership
}

\author{
Michel Le Breton ${ }^{1}$, Juan D. Moreno-Ternero ${ }^{2}$, Alexei Savvateev ${ }^{3}$, Shlomo Weber $^{4}$
}

May 2012

\begin{abstract}
This article studies a model of coalition formation for the joint production (and finance) of public projects, in which agents may belong to multiple coalitions. We show that, if projects are divisible, there always exists a stable (secession-proof) structure, i.e., a structure in which no coalition would reject a proposed arrangement. When projects are indivisible, stable allocations may fail to exist and, for those cases, we resort to the least core in order to estimate the degree of instability. We also examine the compatibility of stability and fairness on metric environments with indivisible projects. To do so, we explore, among other things, the performance of several well-known solutions (such as the Shapley value, the nucleolus, or the Dutta-Ray value) in these environments.

Keywords: Stability, Fairness, Membership, Coalition Formation.
\end{abstract} JEL Classification: C71

\footnotetext{
${ }^{1}$ Université de Toulouse 1, GREMAQ and IDEI, Toulouse, France.

${ }^{2}$ Universidad de Málaga, Spain, Universidad Pablo de Olavide, Seville, Spain, and CORE, Université catholique de Louvain, Belgium.

${ }^{3}$ New Economic School, Moscow, Russia.

${ }^{4}$ Southern Methodist University, USA, and New Economic School, Moscow, Russia. We thank Laurence Wolsey for helpful comments and suggestions. This version has been written while the last author has been Senior Braudei Fellow at the European University Institute in Florence. He also wishes to thank the Grant from the Spanish Ministry of Science and Innovation (ECO2008-01300) for the financial support. The second author also acknowledges financial support from the Spanish Ministry of Science and Innovation (ECO2008-03883) as well as from Junta de Andalucía (P08-SEJ-04154). The first author would like to express his gratitude to M. Goemans and M. Skutella for the numerous discussions on combinatorial optimization and game theory while the three of them were affiliated at CORE.
} 


\section{Introduction}

This paper belongs to the literature on the theory of coalition formation and allocation gains from cooperation across players. In economic and social environments where the players may contemplate a possibility of cooperation, one has to address two sets of questions.

The first one is positive: How large are the gains from cooperation? How to identify coalition structures that yield these gains? Will any such "optimal" coalition structure emerge through the voluntary participation of the players?

The second set of questions is of a normative nature: How the gains from cooperation should be allocated in a stable configuration? How this allocation should be modified according to some criterion of fairness in absence of stability requirement?

While intimately connected, these two sets of questions require separate examination. The positive analysis describes the structure of optimal and/or stable coalition structures. Regarding efficiency, we will consider the classical first-best framework without any limitations or constraints on the transfers of the gains from cooperation, or stated equivalently, we adopt the transferable utility (TU) setting. From that perspective, efficiency amounts to identification of coalition structure(s) maximizing the aggregate surplus. Depending upon the magnitude of the returns from cooperation, the optimal coalition structure patterns can display different features: a unique (grand) coalition when the TU cooperative game is super-additive, several "large" coalitions when the TU cooperative game, while not super-additive, still exhibits moderate returns to scale, or even a multiplicity of "small" coalitions if the gains from cooperation are low or (a fortiori) negative. Stability, on the other hand, refers to the fact that the coalition structure and the proposed sharing arrangement of the gains are immune to potential deviations. In this paper, a potential deviation of a coalition will materialize if, acting on their own, the coalition members would be better off than under the proposed arrangement. However, the stability requirement, called secession-proofness which is related to the notion of core in the cooperative game theory, is very demanding, and, indeed, the set of stable coalition structures is often empty. In that case, it is of interest to characterize the set of allocations which in some sense are closest to being secession-proof and to derive a "measure" of the deviation from secession-proofness. In this paper, we will focus on the concept of least core proposed by Maschler, Shapley and Peleg (1979). To explain the concept, suppose, that, in absence of the core, each coalition must be submitted to a penalty for seceding from the grand coalition. 
Then the least core would consist of allocations associated with the minimal penalty that yields secession-proofness. The least core contains (and often coincides with) the nucleolus (Schmeidler (1969)).

The second set of questions refers to issues which are normative. Independently of any stability considerations, we may examine recommendations according to some notion of fairness discussed and characterized in the existing literature. These include the nucleolus, the Shapley value (Shapley (1953)), and the Dutta-Ray value (Dutta and Ray (1989)), among others. While combining positive and normative approaches, we will examine whether some of these equity concerns are compatible with the secessionproofness property. Is it conceivable that stability implies per se some form of fairness, or does a subset of secession-proof allocations satisfy some fairness properties? Alternatively, is there a fundamental conflict between secession-proofness and fairness?

In this paper we address these questions in the context of the following cooperative environment. A group of players contemplates the possibility of creating coalitions to jointly produce and finance a public group project. For the sake of interpretation, it is convenient to view a public project as a conventional public good targeted for specific needs: health (hospital), education (school), sport activities (stadium, gym, swimming pool), cultural activities (theater, museum, library), financial/postal services (bank, post office), etc. We use the term "public" to reflect the absence of congestion effects, i.e., there is no rivalry in consumption among users. The gains from cooperation simply arise from the fact that, as the quality of the service is not reduced by the presence of additional users, it is always beneficial to increase the size of the group. On the other hand, while projects target the same need, they still differ according to some exogenous "horizontal" characteristic. The latter, evaluated by individuals in different ways, would represent heterogeneity in our model. Preference heterogeneity will be described by one or several parameters; we will refer to this space of parameters as the space of heterogeneity. A prominent example that we will use as our key illustration is location. Public projects could be similar in all respects, except for the spatial dimension: assuming that users minimize the transportation costs incurred by the consumption of the service, they will rank public projects according to the distance between their own residence and projects' location.

In Section 2 we consider a setting with divisible public projects, whose scale can vary in a continuous manner. Every individual has a unit demand for the service while exhibiting her own preferences regarding the projects' characteristics. It is traditionally assumed that every individual belongs to 
a unique coalition identified by a public project and its scale. This implies a one-to-one relationship between the set of membership structures and the set of partitions. We depart from this structure by allowing the unit demand to be satisfied by several coalitions: an individual may split her unit demand among different communities. We assume that the cost of operating a community, i.e., implementing a public project, is proportional to the scale of operation. A membership structure is now described by two items:

- a vector describing the project scale in every formed community;

- a matrix describing the allocation of the total membership of each individual across the different communities, consistent with the project scale in these communities.

We then explore the set of secession-proof allocations when each formed community allocates the operating costs to its members. The main result of this section asserts the existence of secession-proof allocations. As a byproduct of the proof, we show that there is a one-to-one relationship between the set of secession-proof allocations and the set of solutions of the dual of a linear program describing the social planner problem. The dual variables correspond to Lindahl prices allowing the full decentralization of core allocations.

In Section 3 we turn to indivisible public projects. We assume that each project is assigned a fixed scale of operation (normalized to one). This setting exhibits a degree of non-convexity due to the fact that the decision of whether to initiate a public project becomes integer-valued: it attains values 0 or 1 only. Without loss of generality, we assume that every individual is member of a single coalition. The introduction of indivisibilities amounts to adding integer constraints in the linear programming formulation, and, as a result, secession-proof allocations may fail to exist. In fact, there is a secession-proof allocation only if there is no integrality gap and the replacement of integer constraints by linear ones does not impact the solution. In Subsection 3.1, we consider a general class of metric environments where individuals and projects are identified by their locations with the associated metric of distances. We show that secession-proof allocations do not, in general, exist and focus our analysis on the least core and the nucleolus. In Subsection 3.2, we consider the special but important case where the heterogeneity space is one-dimensional and individuals' preferences are single-peaked. This setting yields no integrality gap, and, hence, generates the existence of secession-proof allocations. We will then characterize the nucleolus and examine its properties. In particular, we address the issue of equalization of players' payoffs generated by transfers between individuals. Le Breton and Weber (2003) show that a partial equalization, when the gap 
between "advantaged" and "disadvantaged" individuals is reduced but not completely eliminated, is compatible with secession-proofness, whereas full equalization and no equalization are, in general, not. We then examine a degree of equalization imposed by the nucleolus and other solutions.

Section 4 contains the concluding remarks and the description of some open questions related to this line of research.

The Appendix is partitioned into three parts. In the first one (A1) we introduce and discuss various notions of $\varepsilon$ - and least cores, as well as that of the nucleolus. The second (A2) is devoted to the introduction and some results on egalitarian properties of the Dutta-Ray value in our context. Finally, the last part of the Appendix (A3) contains the derivation of the nucleolus in the one-dimensional version of our metric game.

\section{Related Literature}

This paper belongs to the strand of literature that uses a linear programming approach to explore the core and the set of equilibria in an economic environment. Thus, it lies at the intersection of these different areas and contributions.

First, we mention a class of linear production games, introduced by Owen (1975), who expanded the class of assignment games (Shapley and Shubik (1971)). In linear production games individuals are endowed with a bundle of inputs from which outputs are produced under linear constraints. The worth of a coalition is the maximal value of the outputs that can be produced by the coalition. Cooperative games with side payments arising from such linear optimization problems were called LP games by Samet and Zemel (1984). As they show, dual payoffs can be constructed from any dual optimal solution. While the core always contains the set of dual payoffs, in general, the sets are not identical. Samet and Zemel examine conditions for the equivalence of two sets. They also demonstrate that the two sets coincide asymptotically if the set of individuals is replicated many times.

The relationship between the existence of a core under integer constraints and the integrality gap has also been investigated in other areas of the literature including markets and multi-item auctions (e.g., Bichchandani and Mamer (1997), Bichchandani and Ostroy (2002), Kelso and Crawford (1982), Makowski and Ostroy (2000)). When the individuals are assigned to a single coalition, the problem studied in this paper is the well documented as $U n$ capacited facility location problem which has been extensively investigated in operations research. The main focus is on the determination of efficient algorithms and heuristics to deal with the complexities arising from integer constraints. Few papers have explored the question of the cost sharing 
scheme associated with the optimal solution. The more important contributions are due to Kolen (1983), Tamir (1992) and Goemans and Skutella (2004). Kolen was the first to point out the connection between the linear program identifying the core and the dual of the linear program of the social planner. Tamir uses a similar argument in a specific metric environment, whereas Goemans and Skutella present several generalizations of the previous arguments.

In our paper we extensively use the concept of nucleolus and derived it in several special cases. One has to note that the computation of the nucleolus is a notoriously complicated task. It has been calculated for some classes of cooperative games arising in various environments. Those include bankruptcy problems (Aumann and Maschler (1985)), sharing of airport costs (Littlechild (1974), Owen (1974)) and weighted majority games (Peleg (1968)), among others.

\section{The Model with Divisible Public Projects: Gen- eral Existence of the Core}

The environment $\mathcal{E}$ that we consider is described by a 5 -tuple $\{N, M, D, C, F\}$ where $N=\{1, \ldots, n\}$ is a finite set of individuals, $M=\{1, \ldots, m\}$ is a finite set of public projects, $D \equiv\left(d_{1}, \ldots, d_{n}\right)$ is a $n$-dimensional nonnegative vector, $C=\left(c_{i j}\right)_{1 \leq i \leq n, 1 \leq j \leq m}$ is a $n \times m$ non-negative matrix and $F \equiv\left(f_{1}, \ldots, f_{m}\right)$ is a $m$-dimensional non-negative vector representing the unitary costs of the different public projects, where the cost of project $j$ with the scale $y_{j}$ is $f_{j} y_{j}$. Public projects are described by a number of characteristics: they are excludable (any individual can be excluded, at no cost, from the access to a project), but are not subject to congestion (an increase in the number of individuals consuming the project does not reduce the quality of the project). The vector $D$ describes the aggregate demand for public projects for all individuals. For this formulation to be meaningful, it is implicit that public projects are substitutes in the sense that they target the same needs. For instance, all of them are either hospitals, libraries, post offices, or banks. However, while matching a specific economic or social activity, they differ according to a variety of dimensions (location, quality, architecture, type of programs, design of the buildings, opening hours, etc). We will discuss in the concluding section, the issues arising from the consideration of "truly" different public projects.

A physical allocation is described by a 2-tuple $\{X, y\}$ where $X=$ $\left(x_{i j}\right)_{1 \leq i \leq n, 1 \leq j \leq m}$ is a $n \times m$ non-negative matrix and $y \equiv\left(y_{1}, \ldots, y_{m}\right)$ is 
a $m$-dimensional nonnegative vector. It is feasible if $\sum_{1 \leq j \leq m} x_{i j} \geq d_{i}$ for all $i=1, \ldots, n$ and $x_{i j} \leq y_{j}$ for all $i=1, \ldots, n$ and for all $j=1, \ldots, m$. In contrast to the classical literature on coalition structures, we allow here for multiple membership and an individual could have membership in multiple communities: $\frac{x_{i j}}{d_{i}}$ represents the fraction of the total demand of individual supplied by community $j$. Thus, the communities are not necessarily disjoint. Hereafter, for all $j=1, \ldots, m$, we will refer to the group $S_{j} \equiv\left\{i \in N: x_{i j}>0\right\}$ as the community (club, jurisdiction) $j$. The matrix $X$ describes therefore the structure of membership and the fractional assignment of the individuals to the projects. In addition to its membership, a community $j$ is also described by its size or scale of operation, $y_{j}$. Feasibility requires that the demand of any member of the club cannot exceed the "supply" of the club.

Suppose, for example, that the projects are public swimming pools located in a given urban area and that each individual $i$ swims a fixed number of hours $d_{i}$ per week. Then, the matrix simply describes how the total amount of swimming time of each individual is shared across the different swimming pools. If we denote by $y_{j}$ the number of opening hours of the swimming pool $j$, then the time spent in swimming pool $j$ obviously cannot exceed $y_{j}$.

While the public projects serve the same basic need, they are not perfect substitutes. We assume that the gross payoff of individual $i$ from consuming a quantity $x_{i j}$ of project $j$ is $x_{i j}\left(v_{i}-c_{i j}\right)$ where $v_{i}$ is set to be a very large number. The constant benefit part will play no role in the analysis and will be deleted hereafter ${ }^{1}$. The vector $\left(c_{i j}\right)_{1 \leq j \leq m}$ can be interpreted as the vector of the unitary individual costs incurred by individual $i$ for different projects. The cost is linear in $x_{i j}$ and must be therefore interpreted as a variable cost. In the swimming pool example, if $d_{i}$ is the total time spent in swimming during a given period and if each visit lasts one unit of time, then $x_{i j}$ is simply the number of visits to the swimming pool $j$. While deriving a benefit from consuming public projects, individuals incur costs (taxes) of contribution to projects. If $t_{i}$ denotes the total amount of taxes payed by

\footnotetext{
${ }^{1}$ Recall that the demand is inelastic and is not responsive to taxes. One could rationalize the inelastic demand by considering a piecewise linear benefit function of the public good consisting of two components: one $\left[0, d_{i}\right]$ with a very steep slope $v_{i}$ as long as the aggregate consumption is less $d_{i}$, and another $\left[d_{i},+\infty\right.$ [ with a very flat slope whenever the aggregate consumption exceeds $d_{i}$. As long as prices vary in a bounded interval, individual $i$ will select the quantity $d_{i}$.
} 
individual $i$, then her net payoff is equal to

$$
\sum_{1 \leq j \leq m} x_{i j}\left(v_{i}-c_{i j}\right)-t_{i}=v_{i} d_{i}-\left[\sum_{1 \leq j \leq m} c_{i j} x_{i j}+t_{i}\right] .
$$

Without loss of generality, we will assume that $d_{i}=1$ for all $i \in N .^{2}$ Given an environment $\mathcal{E}=\{N, M, C, F\}$, the minimum aggregate cost $^{3}$ is the value $C(N, \mathcal{E})$ of the following linear program $(\boldsymbol{L})$ :

$$
\operatorname{Min} \sum_{i=1}^{n} \sum_{j=1}^{m} c_{i j} x_{i j}+\sum_{j=1}^{m} f_{j} y_{j}
$$

such that $\sum_{j=1}^{m} x_{i j} \geq 1$ for all $i=1, \ldots, n$

$$
\begin{gathered}
x_{i j} \leq y_{j} \text { for all } i=1, \ldots, n \text { and } j=1, \ldots, m \\
x_{i j} \geq 0 \text { for all } i=1, \ldots, n \text { and } j=1, \ldots, m
\end{gathered}
$$

Let $\left(X^{*}, y^{*}\right)=\left(\left(x_{i j}^{*}\right)_{1 \leq i \leq n, 1 \leq j \leq m},\left(y_{j}^{*}\right)_{1 \leq j \leq m}\right)$ be an optimal allocation, i.e., a solution of the program above. It is obvious that the constraints (2) are binding. Note that the second component in the aggregate cost $C(N, \mathcal{E})=$ $\sum_{i=1}^{n} \sum_{j=1}^{m} c_{i j} x_{i j}^{*}+\sum_{j=1}^{m} f_{j} y_{j}^{*}$ describes the monetary cost incurred by the decisions made with regard to different public projects. This depends upon the scale chosen for each such project. Note that no scale larger than 1 will

\footnotetext{
${ }^{2}$ As long as we can fraction the demand of each individual, we can increase the number of customers to have a problem with unitary demand for each of them. More precisely, if $d_{i}$ is an integer different to 1 , as long as we can fraction the demand, it is as if we had $d_{i}-1$ new players (identical to player $i$ ) all with a unitary demand. If we are forced, however, to treat $d_{i}$ as a block, such argument does not work. Take, for instance, three nodes on a line such that the two extreme nodes are equidistant from the center and the distance is equal to 1 . Extreme individuals have a demand equal to 1 and the median individual has a demand equal to 2 . The facility can be constructed at the cost of $f$ in the extremes but not in the center. If the demand of 2 can be divided into 2 units, the total cost is $2 f+2$ ( 2 facilities of size 1 each) or a single facility of size 2 leading to a cost of $2 f+4$. If the demand of 2 must be treated as a block then the cost is $2 f+4$ (if one facility) or $3 f+2$ (if two facilities).

${ }^{3}$ In this quasi linear setting there is a complete equivalence between the set of Pareto optimal allocations and the set of allocations meeting the demand constraints and minimizing the aggregate cost.
} 
be selected as the aggregate demand of any individual has been normalized to 1 . The vector of taxes $T \equiv\left(t_{1}, \ldots, t_{n}\right)$ must balance the budget:

$$
\sum_{i=1}^{n} t_{i}=\sum_{j=1}^{m} f_{j} y_{j}^{*}
$$

An allocation is a 3 -tuple $\{\{X, y\}, t\}$ where $\{X, y\}$ is physical allocation and $t$ is a $n$-dimensional vector of taxes. It is feasible if $\{X, y\}$ is feasible and the budget is balanced. The total cost incurred by individual $i \in N$ in such allocation is:

$$
T_{i} \equiv \sum_{j=1}^{m} c_{i j} x_{i j}+t_{i}
$$

If instead of the entire society $N$, we consider a subset $S \subseteq N$, the minimum aggregate cost affordable to $S$ is the value $C(S, \mathcal{E})$ of the following linear program:

$$
\begin{gathered}
\underset{(X, y)}{\operatorname{Min}} \sum_{i \in S} \sum_{j=1}^{m} c_{i j} x_{i j}+\sum_{j=1}^{m} f_{j} y_{j} \\
\text { such that } \sum_{j=1}^{m} x_{i j} \geq 1 \text { for all } i \in S \\
x_{i j} \leq y_{j} \text { for all } i \in S \text { and } j=1, \ldots, m \\
x_{i j} \geq 0 \text { for all } i \in S \text { and } j=1, \ldots, m,
\end{gathered}
$$

where $X=\left(x_{i j}\right)_{i \in S, 1<j<m}$ is a $(\# S) \times m$ non-negative matrix and $y \equiv$ $\left(y_{1}, \ldots, y_{m}\right)$ is a $m$-dimensional non-negative vector. In a setting where the allocation $\{\{X, y\}, t\}$ is secession-proof, the value $C(S, \mathcal{E})$ represents the stand-alone aggregate cost of group $S$. It is, in fact, the upper bound on the aggregate cost that can be charged to $S$. Formally, an allocation $\{\{X, y\}, t\}$ is secession-proof if

$$
\sum_{i \in S}\left(\sum_{j=1}^{m} c_{i j} x_{i j}+t_{i}\right) \leq C(S, \mathcal{E}) \text { for all } S \subseteq N .
$$

It is easy to see that in the space of individual payoffs, there is a oneto-one relationship between the set of secession-proof allocations and the core of the cooperative game with transferable utility, where the value of the characteristic function assigned to a coalition $S$ is $C(S, \mathcal{E})$. Proposition 
1 below asserts that for any environment $\mathcal{E}$, there always exist secessionproof allocations. In fact, there is a one-to-one relationship between the set of secession-proof allocations and the optimal solutions of the dual of the linear program $(\boldsymbol{L})$. The dual linear program $(\boldsymbol{D} \boldsymbol{L})$ is presented as follows:

$$
\begin{gathered}
\underset{(T, P)}{\operatorname{Max}} \sum_{i=1}^{n} T_{i} \\
\text { such that } \sum_{i=1}^{n} p_{i j} \leq f_{j} \text { for all } j=1, \ldots, m \\
-p_{i j}+T_{i} \leq c_{i j} \text { for all } i \in N \text { and } j=1, \ldots, m \\
p_{i j} \geq 0 \text { for all } i \in N \text { and } j=1, \ldots, m,
\end{gathered}
$$

where $P=\left(p_{i j}\right)_{1 \leq i \leq n, 1 \leq j \leq m}$ is a $n \times m$ non-negative matrix and $T \equiv$ $\left(T_{i}\right)_{1 \leq i \leq n}$ is a $n$-dimensional non-negative vector. We denote by $D(N, \mathcal{E})$ the value of dual linear program $(\boldsymbol{D L})$ so described. Let

$$
\left(P^{*}, T^{*}\right)=\left(\left(p_{i j}^{*}\right)_{1 \leq i \leq n, 1 \leq j \leq m},\left(T_{i}^{*}\right)_{1 \leq i \leq n}\right)
$$

be an optimal solution of program $(\boldsymbol{D L})$ and let

$$
t_{i}^{*}=\sum_{i=1}^{n} p_{i j}^{*} x_{i j}^{*} \text { for all } i \in N .
$$

We claim that the allocation $\left\{\left\{X^{*}, y^{*}\right\}, t^{*}\right\}$ is secession-proof. Let $S$ be a proper subset of N: $S \varsubsetneqq N$. We will prove that

$$
\sum_{i \in S} T_{i}^{*} \leq C(S, \mathcal{E})
$$

which amounts to show that

$$
\sum_{i \in S} T_{i}^{*} \leq \sum_{i \in S} \sum_{j=1}^{m} c_{i j} x_{i j}+\sum_{j=1}^{m} f_{j} y_{j}
$$

for all $\{X, y\}$ satisfying inequalities (6), (7) and (8). From (6) and (10), we deduce

$$
\sum_{i \in S} \sum_{j=1}^{m} c_{i j} x_{i j}+\sum_{j=1}^{m} f_{j} y_{j} \geq \sum_{i \in S} \sum_{j=1}^{m}\left(T_{i}^{*}-p_{i j}^{*}\right) x_{i j}+\sum_{j=1}^{m} f_{j} y_{j}=\sum_{i \in S} T_{i}^{*}+\sum_{j=1}^{m}\left(f_{j} y_{j}-\sum_{i \in S} p_{i j}^{*} x_{i j}\right)
$$


Then, from (7), (9) and (11), we obtain

$$
\sum_{j=1}^{m}\left(f_{j} y_{j}-\sum_{i \in S} p_{i j}^{*} x_{i j}\right) \geq \sum_{j=1}^{m}\left(\sum_{i \in N} p_{i j}^{*} y_{j}-\sum_{i \in S} p_{i j}^{*} x_{i j}\right) \geq \sum_{j=1}^{m}\left(\sum_{i \in S} p_{i j}^{*} y_{j}-\sum_{i \in S} p_{i j}^{*} x_{i j}\right) \geq 0
$$

The inequality (12) follows immediately. By the duality theorem of linear programming, program $(\boldsymbol{L})$ and program $(\boldsymbol{D} \boldsymbol{L})$ yield the same values. Thus, we have $\sum_{i \in N} T_{i}^{*}=C(N, \mathcal{E})$, which implies that any optimal solution of the dual linear program (DL) is a core imputation generated by a secessionproof allocation.

Now consider an arbitrary secession-proof allocation $\left\{\left\{X^{*}, y^{*}\right\}, t^{*}\right\}$ and let $T^{*}$ be the corresponding core imputation. Let $S_{j}^{*} \equiv\left\{i \in N: T_{i}^{*}-c_{i j}>0\right\}$ and define $p_{i j}^{*}$ as follows

$$
p_{i j}^{*}=\left\{\begin{array}{l}
T_{i}^{*}-c_{i j} \text { if } T_{i}^{*}-c_{i j}>0 \\
0 \text { otherwise }
\end{array}\right.
$$

We claim that $\left(T^{*}, P^{*}\right)$ where $P^{*}=\left(p_{i j}^{*}\right)_{1 \leq i \leq n, 1 \leq j \leq m}$ is an optimal solution of the dual linear program $(\boldsymbol{D L})$. Note first that by construction (10) and (11) hold true. It remains to prove that:

$$
\sum_{i=1}^{n} p_{i j}^{*}=\sum_{i \in S_{j}^{*}} p_{i j}^{*} \leq f_{j} \text { for all } j=1, \ldots, m
$$

Assume, on the contrary, that for some $j, \sum_{i \in S_{j}^{*}} p_{i j}^{*}>f_{j}$. This implies $\sum_{i \in S_{j}^{*}} T_{i}^{*}>\sum_{i \in S_{j}^{*}} c_{i j}+f_{j}$, and therefore that the coalition $S_{j}^{*}$ would benefit from secession, a contradiction to our assumption.

We have proved the following result:

Proposition 1 For any environment E, the set of secession-proof allocations is non empty and it coincides with the set of solutions of the dual linear program $(\boldsymbol{D L})$.

As we indicated above, our paper lies at the intersection of different research areas, and some of the contributions cited in the introduction should receive the credit for several of the arguments used here. Our proof is self contained and quite simple, as it utilizes quite elementary tools from linear programming. 
- The matrix of dual variables $P^{*}=\left(p_{i j}^{*}\right)_{1 \leq i \leq n, 1 \leq j \leq m}$ has a nice and important interpretation. It is a matrix of Lindahl prices as there is a price for each pair composed of a public good and a public project. To some extent, the proposition above asserting the existence of the core, in fact, yields the existence of Lindahl equilibria in this quasi-linear economy with $m$ public goods, one private good and a constant returns to scale technology. A similar statement appears in Mas-Colell (1980). The optimal behavioral responses of individuals to these prices provide a decentralization device for the allocation. While this result could be derived from more general existence results on Lindahl equilibria under convexity assumptions, the direct and simple proof provided here is however instructive per se. The prices also display some interesting features. From the complementary slackness conditions, we observe that

$$
\text { If } x_{i j}^{*}>0 \text {, then } p_{i j}^{*}=T_{i}^{*}-c_{i j} \text { and if } p_{i j}^{*}>0 \text {, then } x_{i j}^{*}=y_{j}^{*} \text {. }
$$

For each $i \in N$, let $S_{i}^{* *} \equiv\left\{j \in M: x_{i j}^{*}>0\right\}$ be the list of communities containing $i$ as a member. From the first condition, we obtain the constant total unit cost for $i$ to get access to any of these communities. This is of course the only way to guarantee decentralization. If it were violated, some of these communities, say, $j$, would be avoided by $i$, contradicting our assumption of positive $x_{i j}^{*}$. The price equality is interesting as it entails some form of partial equalization across communities. From the second condition, we deduce the standard marginal cost pricing rule demanding that a customer is charged a positive price for her consumption only if the supply constraint is binding.

- Note that access prices to a specific community $j$ may entail some discrimination across individuals. It is not difficult to construct examples of nonexistence under the second best constraint imposing price uniformity across customers.

- It is not necessarily the case that individual $i$ is indifferent between the projects in $S_{j}^{* *}$ given the costs $c_{i j}$. What is demonstrated above is that the second component of the cost compensates for the differentials across the $c_{i j}$.

- The solutions of the primal linear program (L) represent the set of Pareto optimal physical allocations. The vector $y^{*}$ describes not only the list of selected projects (the public project $j$ is selected if $y_{j}^{*}>0$ ), but also the scale of each of them. In contrast to the traditional framework, a community is described by its (horizontal) type $j \in M$ and size $y_{j}^{*}$. As 
already explained, the matrix describes the structure of membership across individuals and communities together with their membership shares.

\section{The Model with Indivisible Public Projects}

While the divisible setting of the previous section yields a general existence result on the set of secession-proof allocations, in this section we explore the difficulties imposed by integer constraints associated with the traditional framework. More precisely, assume now that all the physical variables are binary: the project is either selected or not, and each individual is member of a single community. A physical outcome consists of a partition of the population $N$ into $k$ communities (with $k \leq m$ ) with a distinct public project adopted by each community. The introduction of these constraints transforms the original linear program $(\mathbf{L})$ into the following integer linear $\operatorname{program}^{4}(\widehat{\mathbf{L}})$.

$$
\begin{gathered}
\underset{(X, y)}{\operatorname{Min}} \sum_{i=1}^{n} \sum_{j=1}^{m} c_{i j} x_{i j}+\sum_{j=1}^{m} f_{j} y_{j} \\
\text { such that } \sum_{j=1}^{m} x_{i j} \geq 1 \text { for all } i=1, \ldots, n \\
x_{i j} \leq y_{j} \text { for all } i=1, \ldots, n \text { and } j=1, \ldots, m \\
x_{i j} \in\{0,1\} \text { for all } i=1, \ldots, n \text { and } j=1, \ldots, m \\
y_{j} \in\{0,1\} \text { for all } j=1, \ldots, m .
\end{gathered}
$$

This type of integer linear programs is notoriously difficult to solve and known to be computationally hard. Algorithms with a guaranteed performance level, satisfying some computational constraints, have been developed in the field of operations research, where the above problem has been studied very extensively. ${ }^{5}$ It has been called Uncapacited Facility Location Problem (UFLP) (Cornuéjols, Nemhauser and Wolsey (1990)) due to the most celebrated application of this setting of optimal location of facilities and the assignment of customers to formed facilities. The set $M$ corresponds to the potential facilities sites (in the geographical space) and the set $N$ corresponds to the actual locations of customers. If the geographical space is $\mathbb{R}^{2}$,

\footnotetext{
${ }^{4}$ Balinski (1965) first introduced this integer linear programming formulation of the uncapacited facility location problem.

${ }^{5}$ See Mirchandani and Francis (1990) for an overview of the state of the arts and ReVelle, Eiselt and Daskin (2008) for a recent selective bibliography.
} 
$M$ is a $m$-tuple $\left\{q_{j}\right\}_{1 \leq j \leq m}$ of vectors in $\mathbb{R}^{2}$ and $N$ is a $n$-tuple $\left\{p_{i}\right\}_{1 \leq i \leq n}$ of vectors in $\mathbb{R}^{2}$. Further, it is assumed that there exists a non-decreasing function $h$ such that the $\operatorname{costs} c_{i j}$ are equal to $h\left(d\left(p_{i}, q_{j}\right)\right)$, where $d$ is a distance on $\mathbb{R}^{2}$ (e.g., Euclidean, rectilinear, Manhattan) for all $i=1, \ldots, n$ and $j=1, \ldots, m$. The cost $c_{i j}$ describes the transportation cost that would be incurred by a customer located in $p_{i}$ to consume a service located in $q_{j}$. It is natural to assume that such costs are non-decreasing with respect to some appropriate distance. The term uncapacited refers to the public aspect of the good - there is no limit on the number of customers assigned to a given facility.

We will denote by $\widehat{C}(N, \mathcal{E})$ the value of the integer linear program $(\widehat{\mathbf{L}})$. As the linear program $(\mathbf{L})$ arises through a linear relaxation of the integer constraints (13) and (14) in the program $(\widehat{\mathbf{L}})$, we have

$$
c(N, \mathcal{E}) \equiv \frac{C(N, \mathcal{E})}{\widehat{C}(N, \mathcal{E})} \leq 1
$$

A careful examination of the argument presented in Section 2 shows that $C(N, \mathcal{E})$ is the value of the following linear program

$$
\begin{gathered}
\operatorname{Max}_{T} \sum_{i=1}^{n} T_{i} \\
\text { such that } \sum_{i \in S} T_{i} \leq \widehat{C}(S, \mathcal{E}) \text { for all } S \subseteq N,
\end{gathered}
$$

where $\widehat{C}(S, \mathcal{E})$ has been defined similarly to $C(S, \mathcal{E})$, except that the linear constraints $x_{i j} \geq 0$ for all $i \in S$ and $j=1, \ldots, m$ have been replaced by the integer constraints $x_{i j} \in\{0,1\}$ for all $i \in S$ and $j=1, \ldots, m$. We deduce from this simple but important fact that the maximal amount of money that can be collected without violating the secession-proofness constraints is equal to $C(N, \mathcal{E})$. Two cases may arise:

$C(N, \mathcal{E})=\widehat{C}(N, \mathcal{E})$ or $c(N, \mathcal{E})=1$. In such case, the total aggregate cost has been collected without violation of secession-proofness and the core is nonempty.

$C(N, \mathcal{E})<\widehat{C}(N, \mathcal{E})$ or $c(N, \mathcal{E})<1$. In such case, it is impossible to recover the total aggregate cost under the secession-proofness requirement. Only the ratio $c(N, \mathcal{E})$ can be recovered. If this number is sufficiently large, the problem of budget deficit can, in some cases, be solved by an external source. From this perspective, the closer to 1 this ratio is, the more "stable" the environment is. 
The second case corresponds to what is called an "integrality gap" in integer linear programming. In the case where multiple memberships are prohibited, the above argument shows that the set of secession-proof allocations is nonempty only in the absence of the integrality gap.

Goemans ans Skutella (2004) have considered a broader family of facility location problems: it contains the UFLP as a special case and proved that for any member of this family the cost allocation problem is equivalent to the dual of the LP relaxation of the facility location problem. This implies that the core is non-empty if and only if there is no integrality gap for the LP relaxation of the cost allocation problem. The family of problems that they consider is broad enough to accomodate many additional constraints on facility like, for instance, capacity, quota or incompatibility constraints.

\subsection{Metric Environments: The Least Core}

We will now examine a subclass of environments called metric environments, that covers many important applications. We wish to point out that for any such environment $\mathcal{E}$, the integrality gap is "universally" bounded away from 0 , i.e., the ratio $c(N, \mathcal{E})$ exceeds some given value $\alpha$ irrespective of the specific identification of $\mathcal{E}$.

Before providing a formal statement of the result, it is useful to offer an example illustrating basic differences between the continuous and integer settings. Consider the location environment $\mathcal{E}$ in $\mathbb{R}^{2}$ with with four individuals and four projects, where each individual shares the location with one project. Let $p_{1}=q_{1}=(0,0), p_{2}=q_{2}=(1,0), p_{3}=q_{3}=(1,1)$ and $p_{4}=q_{4}=(0,1)$ and the distances are given by:

$$
d_{i j} \equiv d\left(p_{i}, q_{j}\right)= \begin{cases}0 \text { if } j=i \\ 1 \text { if } j=i+1 \text { or } j=i+3 & (\bmod 4) \\ 2 \text { if } j=i+2 & (\bmod 4)\end{cases}
$$

Also, the costs $c_{i j}$ are equal to the distances $d_{i j}$ and $f_{j}=f>0$ for all $j=1,2,3,4$. Let us calculate the values $C(N, \mathcal{E})$ and $\widehat{C}(N, \mathcal{E})$. The simple algebra shows that

$$
\widehat{C}(N, \mathcal{E})=\left\{\begin{array}{l}
4 f \text { if } f \in[0,1] \\
2 f+2 \text { if } f \in[1,2] \\
f+4 \text { if } f \in[2,+\infty[
\end{array}\right.
$$

Depending upon the value of the parameter $f$, it is optimal to "open" either 1, 2 or 4 facilities. ${ }^{6}$ For the sake of numerical illustration, let us assume that

\footnotetext{
${ }^{6}$ The case of 3 facilities occurs also when $f=1$.
} 
$f=2$. Then $\widehat{C}(N, \mathcal{E})=6$.

To derive the value of $C(N, \mathcal{E})$, let us define the set-function $D$ on the set of nonempty proper subsets of $N$. Namely, let

$$
D(S)=\left\{\begin{array}{lll}
4 & \text { if } & s=3 \\
4 & \text { if } & S=\{1,3\}, \text { or }\{2,4\} \\
3 & \text { if } & S=\{1,2\},\{2,3\},\{3,4\}, \text { or }\{4,1\} \\
2 & \text { if } \quad s=1
\end{array}\right.
$$

where $s$ is the cardinality of the set $S$.

It is easy to see that $C(N, \mathcal{E})$ is the value of the following program $(\mathbf{V})$ :

$$
\underset{T}{\operatorname{Max}} \sum_{i \in N} T_{i} \text { such that } \sum_{i \in S} T_{i} \leq D(S) \text { for all } S \subseteq N .
$$

The program (V) can be rewritten $a^{7}$

$$
\underset{T}{\operatorname{Max}} \sum_{i \in N} T_{i}
$$

$$
\begin{gathered}
\text { such that } \sum_{i \in S} T_{i} \leq 4 \text { for all } S \subseteq N \text { with } s=3 \\
\max \left\{T_{1}+T_{2}, T_{2}+T_{3}, T_{3}+T_{4}, T_{4}+T_{1}\right\} \leq 3 \\
\max \left\{T_{1}, T_{2}, T_{3}, T_{4}\right\} \leq 2 .
\end{gathered}
$$

It is straightforward to show that the vector $\left(\frac{4}{3}, \frac{4}{3}, \frac{4}{3}, \frac{4}{3}\right)$ is the unique optimal solution of this linear program. Therefore, $C(N, \mathcal{E})=\frac{16}{3}$. In this example, the ratio $\frac{C(N, \mathcal{E})}{\widehat{C}(N, \mathcal{E})}$ is equal to $\frac{8}{9} \simeq 89 \%$, a value which is quite close to 1 ! Note that the primal linear program (L) also has a unique solution

$$
y^{*}=\left(\frac{1}{3}, \frac{1}{3}, \frac{1}{3}, \frac{1}{3}\right)
$$

and

$$
X^{*}=\left(\begin{array}{cccc}
\frac{1}{3} & \frac{1}{3} & 0 & \frac{1}{3} \\
\frac{1}{3} & \frac{1}{3} & \frac{1}{3} & 0 \\
0 & \frac{1}{3} & \frac{1}{3} & \frac{1}{3} \\
\frac{1}{3} & 0 & \frac{1}{3} & \frac{1}{3}
\end{array}\right)
$$

Here, the optimal scale of each facility is $\frac{1}{3}$ and the structure of multiple memberships presented by the matrix shows that each individual gets her top

\footnotetext{
${ }^{7}$ Some non-binding constraints are not presented here.
} 
choice for one third of the demand and her second best for the remaining two thirds; she is never assigned to her least preferable facility. The personalized Lindahl prices of individual 1 are $p_{11}=\frac{4}{3}, p_{12}=p_{14}=\frac{1}{3}$.

Note that the program $(\mathbf{V})$ can be restated in terms of balanced sets (Bondareva (1962) and Shapley (1967)) used in the cooperative game theory, A game with transferable utility (TU) is a pair $(N, V)$, where $N=\{1, \ldots, n\}$ with $n \geq 2$ is a finite set of players and $V$ is a function that associates a real number $V(S)$ to each subset $S$ of $N$. A collection of subsets of $N$, $\pi=\left\{S_{1}, S_{2}, \ldots, S_{K}\right\}$ is called balanced if for every $S \in \pi$ there exists a non-negative number $\delta_{S}$, called balancing weight, such that $\sum_{S \in \pi: i \in S} \delta_{S}=1$. for every player $i \in N$. Denote the set of all balanced collections by $\mathcal{B}$. Then the problem (V) amounts to finding $\underset{\pi \in \mathcal{B}}{\operatorname{Max}} \sum_{S \in \pi} \delta_{S} V(S)$.

The examination of the optimal solution of $(\mathbf{L})$ shows that the binding constraints are associated with three-person coalitions and the relevant balanced family of coalitions in $(\mathbf{V})$ is:

$$
\{\{1,2,3\},\{1,2,4\},\{1,3,4\},\{2,3,4\}\}
$$

with the vector $\delta \equiv\left(\frac{1}{3}, \frac{1}{3}, \frac{1}{3}, \frac{1}{3}\right)$ of corresponding balancing weights.

The example yields quite a high value of $c(N, \mathcal{E})$. Is it a pure coincidence? When we let the parameter $f$ vary, we find that

$$
C(N, \mathcal{E})=\left\{\begin{array}{l}
4 f \text { if } f \in[0,1] \\
\frac{4 f+8}{3} \text { if } f \in[1,4] \\
f+4 \text { if } f \in[4,+\infty[
\end{array}\right.
$$

Thus, the minimal value of $c(N, \mathcal{E})$ with respect to $f$ is attained for $f=2$ and is equal to $89 \%$. Quite surprisingly, this ratio is bounded from below whenever the environment $\mathcal{E}$ belongs to the domain of metric environments described below.

Formally, an environment $\mathcal{E}=\{N, M, D, C, F\}$ is called metric if

$$
c_{i k} \leq c_{i j}+c_{j l}+c_{l k} \text { for all } i, l \in N \text { and } j, k \in M
$$

This property holds when the sets $N$ and $M$ are points in a metric space and the $c_{i j}$ are defined as the pairwise distances between these points. ${ }^{8}$ Let $\mathbf{M}$ denote the set of metric environments. Shmoys, Tardos and Aardal (1997)

\footnotetext{
${ }^{8}$ As already pointed out, the uncapacited facility location problem has been extensively studied in operations research, especially, in the field of approximation algorithms. A number of different approximation algorithms have been proposed but we are only in-
} 
were the first to found that if $\mathcal{E} \in \mathbf{M}$, then $c(N, \mathcal{E}) \geq .316$. This universal bound was later refined by Chudak (1998) and Chudak and Shmoys (1999) to $1 /\left(1+\frac{2}{e}\right) \simeq 0.576$, and, more recently, to 0.632 by Sviridenko (2002). The mere existence of this absolute constant in the context of a metric environment, is surprising in itself. On the other hand, Guha and Khuller (1999) have shown that this problem is hard to approximate within a factor better than 0.684 implying that there exist metric environments $\mathcal{E}$ such that $c(N, \mathcal{E}) \leq 0.684$.

We deduce from the above results that if $\mathcal{E} \in \mathbf{M}$, then we can always recover at least $63.2 \%$ of the total cost and at most $68.4 \%$ in some specific metric environments. Let us call a feasible allocation $\left(X^{*}, y^{*}, t^{*}\right)=$ $\left(\left(x_{i j}^{*}\right)_{1 \leq i \leq n, 1 \leq j \leq m},\left(y_{j}^{*}\right)_{1 \leq j \leq m},\left(t_{i}^{*}\right)_{1 \leq i \leq n}\right) \alpha$-secession proof, if

$$
\begin{gathered}
x_{i j}^{*} \in\{0,1\} \text { for all } i=1, \ldots, n \text { and } j=1, \ldots, m \\
\qquad y_{j}^{*} \in\{0,1\} \text { for all } j=1, \ldots, m \\
\sum_{i \in S} \sum_{j=1}^{m} c_{i j} x_{i j}^{*}+\sum_{i \in S} t_{i}^{*} \leq \widehat{C}(S, \mathcal{E}) \text { for all } S \subseteq N \\
\sum_{i \in N} \sum_{j=1}^{m} c_{i j} x_{i j}^{*}+\sum_{i \in N} t_{i}^{*} \geq \alpha \widehat{C}(N, \mathcal{E}) .
\end{gathered}
$$

Of course, if there exist $\alpha$-secession proof allocations, there are $a$-secessionproof allocations for all $a \leq \alpha$. Let $\alpha(\mathcal{E})$ be the largest value of $\alpha$ such that the environment $\mathcal{E}$ admits $\alpha$-secession proof allocations and let $T \equiv$ $\left(T_{i}\right)_{1<i<n}$ be the $n$-dimensional vector induced by any such $\alpha(\mathcal{E})$-secession proof allocation. It satisfies the following list of inequalities:

$$
\sum_{i \in S} T_{i} \leq \widehat{C}(S, \mathcal{E}) \text { for all } S \subseteq N \text { and } \sum_{i \in N} T_{i}=\alpha(\mathcal{E}) \widehat{C}(N, \mathcal{E}) .
$$

terested in those based on the above linear programming relaxation. The motivations are quite different from ours. Solving a linear program is "easy" according to the theory of computational complexity. Therefore, if the relaxation leads to a solution which is not too distant from the solution of the integer linear program, we can use this scheme as an approximation algorithm with the performance guarantee of $c(N, \mathcal{E})$. In fact, this literature usually utilizes the reverse ratio $1 / c(N, \mathcal{E})$. For the sake of consistency, we proceed with our specification. Of course, many other approximation algorithms are not based on LP rounding (Jain et al. (2003), Jain and Vazirani (2001), Madhian, Ye and Zhang (2002)). The current best constant factor is 0.66 . 
Let $\widetilde{T}$ be the vector where $\widetilde{T}_{i}=\frac{T_{i}^{*}}{\alpha(\mathcal{E})}$ for all $i=1, \ldots, n$. The above inequalities translate into

$$
\sum_{i \in S} \widetilde{T}_{i} \leq \frac{1}{\alpha(\mathcal{E})} \widehat{C}(S, \mathcal{E}) \text { for all } S \subseteq N \text { and } \sum_{i \in N} \widetilde{T}_{i}=\widehat{C}(N, \mathcal{E}) .
$$

This shows that $\widetilde{T}$ belongs to $L C_{m}(\mathcal{E})$, the multiplicative least core of the environment $\mathcal{E}$, and that $\left(\frac{1}{\alpha(\mathcal{E})}-1\right)$ is the multiplicative least core value. The following result summarizes the above discussion:

Proposition 2: For any environment $\mathcal{E} \in \mathbf{D}$, the multiplicative least core value is at most equal to 0.5822

\subsection{The One-dimensional Metric Environment: the Core, the Nucleolus and Other Solutions}

In this subsection, we will focus our attention on a one-dimensional special case of the metric model discussed in the preceding section. More precisely, we will consider the environment $\mathcal{E}=\{N, M, D, C, F\}$, where $N=\{1, \ldots, n\}$ is a finite set of individuals that coincides with the set of project locations $M$. We assume that the costs are $c_{i j}=d|i-j|$ and $f_{j}=f$ for all individuals $i$ and projects $j$ where $d$ and $f$ are positive parameters. This particular one-dimensional version of the problem describes the situation where there are $n$ equally populated demand sites located along the line (main street). The distance between two demand sites is constant $(d)$ and the cost of transportation is linear with respect to distance. A public facility can be constructed in any of the demand sites and the set up cost is independent of the location. The environment $\mathcal{E}$ is now described by the triple $\{N, d, f\}$.

Any such environment satisfies the single peakedness condition. ${ }^{9}$ To recall, a domain of environments is single-peaked if there exists an ordering $\sigma$ of the projects such that for each $i \in N$, there exists a project $j(i)$ such that $c_{i j}$ decreases when $j$ varies from $\sigma(1)$ to $\sigma(j(i))$ and increases when $j$ varies from $\sigma(j(i))$ to $\sigma(m)$. For any single-peaked environment, the results by Goemans and Skutella (2004), Kolen (1983), Tamir (1992) and Trubin (1976) guarantee the absence of the integrality gap and, hence, the non-emptiness of the set of secession-proof allocations. Given that the existence of secession-proof allocations is guaranteed, we will examine whether

\footnotetext{
${ }^{9}$ Another domain with this property is the case where the customers and facilities are located on a tree and the distances are calculated along the paths allowed by the tree.
} 
it is possible to combine secession-proofness and some fairness requirements through the selection of an appropriate allocation. As there are many alternative definitions of fairness, we will explore the implications of different popular solutions serving as natural candidates to meet these two objectives.

In this setting, the disutility or transportation cost incurred by an individual located in $t$ and consuming the services of a facility located in $p$, is determined by the distance between $t$ and $p$, i.e., $d|t-p|$. For each $S \subseteq N$, the minimal transportation cost of the citizens of $S$ is given by $\min _{p \in N} \sum_{t \in S} d|t-p|$. Note that, as $S$ is finite, there always exists an optimal location of the facility and, therefore, the previous amount is well defined. It is useful to note that for every set $S$ the total transportation cost is minimized when the project is chosen at the "median user" location $m(S)$, that is determined by the equality $|\{t \in S: t \leq m(S)\}|=\mid\{t \in S: t \geq m(S) \mid$. If $S$ has an odd number of users then its median user is uniquely defined. However, if $S$ consists of an even number of users, the median user is not unique. To avoid ambiguity, we denote by $m(S)$ the left-most median of $S$.

The overall cost of a region $S \subseteq N$ will therefore be the sum of its facility and transportation costs. Formally,

$$
c(S)=f+d \cdot \min _{p \in N} \sum_{t \in S}|t-p| .
$$

Under our assumptions, $c(S)$ can be reformulated as follows. Let $S \subseteq N$ be a given region and $\sigma$ a bijection between $S$ and $\{1,2, \ldots, s\}$, such that $\sigma(k) \leq \sigma(k+1)$ for all $k$. Then,

$$
c(S)=f+\left(\sum_{k=0}^{\left[\frac{s}{2}\right]-1}(\sigma(s-k)-\sigma(k+1))\right) \cdot d,
$$

where $[x]$ denotes the integer part of $x$. In particular, if $s=1$, then $c(S)=f$. If $s=2,3$ then $c(S)=f+\max \{|i-j|: i, j \in S\} \cdot d$. If $s=n$, then

$$
c(S)=f+\left(\sum_{k=1}^{\left[\frac{n}{2}\right]} n-(2 k-1)\right) \cdot d=\left\{\begin{array}{ll}
f+\frac{n^{2}}{4} \cdot d & \text { if } n \text { even } \\
f+\frac{n^{2}-1}{4} \cdot d & \text { if } n \text { odd }
\end{array} .\right.
$$

In this environment, an allocation rule is a mapping that associates with each facility cost $f$ and distance parameter $d$ a cost allocation $\gamma(f, d)=$ $\left(\gamma_{1}(f, d), \ldots, \gamma_{n}(f, d)\right) \in \mathbb{R}_{+}^{n}$. In what follows, we omit $(f, d)$ in description of allocation rules. 
In contrast to the previous part of the paper, we will now focus on environments where the optimal coalition structure is the grand coalition. We refer to this situation as $N$-Efficiency. ${ }^{10}$ Formally,

$N$-Efficiency. An environment $\mathcal{E}=\{N, d, f\}$ is $N$-efficient if

$$
c(N) \leq \sum_{S \in \pi} c(S) \text { for all partitions } \pi \text { of } N
$$

The proposition below identifies necessary and sufficient conditions for $N$-Efficiency:

Proposition 3: The following statements hold:

(i) If $n=4 k$ then $\mathcal{E}$ is $N$-efficient if and only if $f \geq 2 k^{2} d$.

(ii) If $n=4 k+2$ then $\mathcal{E}$ is $N$-efficient if and only if $f \geq \frac{(2 k+1)^{2}+1}{2} d$.

(iii) If $n=4 k+1$ then $\mathcal{E}$ is $N$-efficient if and only if $f \geq k(2 k+1) d$.

(iv) If $n=4 k+3$ then $\mathcal{E}$ is $N$-efficient if and only if $f \geq\left(2 k^{2}+3 k+1\right) d$.

Assume, for instance, that $n=4 k$. If $\mathcal{E}$ is $N$-efficient then

$$
c(N)=f+4 k^{2} d \leq c\left(\{1, \ldots, 2 k\}+c(\{2 k+1, \ldots, n\})=2 f+2 k^{2} d\right.
$$

or, equivalently, $f \geq 2 k^{2} d$, which proves one implication of $(i)$. As for the converse implication, it suffices to note that $\{\{1, \ldots, 2 k\},\{2 k+1, \ldots, n\}\}$ is the "most expensive" partition of $N$. The remaining proofs proceed along the same lines.

$N$-efficiency is a property of cooperative environments where the gains from cooperation are large, as characterized by Proposition 3. An extremely relevant selection of the core is the nucleolus. Thus, the next proposition provides an important instance of secession-proof allocation in this setting. ${ }^{11}$

\footnotetext{
${ }^{10}$ It is not difficult to show that, for sufficiently small values of $f$, it could be optimal to avoid transportation costs upon locating a facility at each node. We, nevertheless, prefer to focus on the case in which the optimal coalition is the grand coalition and hence the notion of N-efficiency, which, de facto, is excluding low values of $f$, as we see in Proposition 3.

${ }^{11}$ As shown in Appendix A3, we prove the statement of Proposition 2 for $n \leq 6$. We conjecture that the statement is also true for all $n \geq 7$.
} 
Proposition 4: If the environment $\mathcal{E}$ is $N$-efficient and if $n \leq 6^{12}$, there exists a threshold $f_{0}$ such that the nucleolus of the cost-sharing game described above is given by the allocation rule $\gamma$, where:

- If $n$ is even, $\gamma=\frac{f}{n}(1, \ldots, 1)+d\left(\frac{n-1}{2}, \frac{n-3}{2}, \ldots, \frac{3}{2}, \frac{1}{2}, \frac{1}{2}, \frac{3}{2}, \ldots, \frac{n-3}{2}, \frac{n-1}{2}\right)$.

- If $n$ is odd and $f \leq f_{0}$ $\gamma=\frac{f}{n-1}(1, \ldots, 1,0,1, \ldots, 1)+d(\alpha(n),(\alpha(n)-1), \ldots, 1, \ldots,(\alpha(n)-1), \alpha(n))$, where

$$
\alpha(n)=\left(\frac{n-1}{2}-\frac{1}{n-1}\right)
$$

- If $n$ is odd and $f \geq f_{0}$

$$
\gamma=\frac{f}{n}(1, \ldots, 1)+d\left(\frac{n-1}{2}, \frac{n-3}{2}, \ldots, 1,0,1, \ldots, \frac{n-3}{2}, \frac{n-1}{2}\right) .
$$

Note that when $n$ is even, the allocation consists in dividing equally the facility cost and letting every user to pay for its transportation cost. The case where $n$ is odd is more interesting. If the economies of scale are large enough, then the solution is the same as in the case where $n$ is even. But if the economies of scale are large enough to justify the formation of the grand coalition but still below some critical threshold, then the nucleolus is a more intricate solution.

It is well known that the Shapley value, the best-known solution for cost-sharing games, is only a selection of the core for certain domains. This is not one of them. In order to show this, it suffices to consider the case in which $n=3$. It is not difficult to see that the Shapley value in that case would be given by:

$$
\left(\frac{2 f+5 d}{6}, \frac{f+d}{3}, \frac{2 f+5 d}{6}\right)
$$

Thus, if $d \leq f \leq \frac{5}{4} d$, the environment would be $N$-efficient and the allocation prone to secession. Therefore, there exist $N$-efficient environments for which the Shapley value is not a secession-proof allocation.

\footnotetext{
${ }^{12}$ This is shown in Appendix A3. We conjecture that the statement is also true for all $n \geq 7$.
} 
Within the set of secession-proof allocations for such environments, we would like to identify the other allocations which can be considered to be fair. The task is delicate as there is not a single uncontroversial definition of fairness. Some of these notions take into account the stand alone cost $c(S)$ of every coalition $S$ of users so that the strength or legitimate aspirations of members of coalition $S$, given by $c(S)$, have to be taken into account. In cooperative game theory, many popular solutions, including the nucleolus, the Shapley value and the Dutta-Ray egalitarian solution belong to this family. Some other notions ignore this "reservation value" and aim to equalize as much as possible the utilities of the users irrespective of their positions in the game. In what follows we discuss the properties of solutions based on these two approaches.

The most popular (partial) ordering of allocations according to fairness is the so-called Lorenz ordering $\succsim_{L}$. Given $x, y \in \mathbb{R}^{n}$ satisfying $x_{1} \leq \ldots \leq x_{n}$, $y_{1} \leq \ldots \leq y_{n}$, and $\sum_{i=1}^{n} x_{i}=\sum_{i=1}^{n} y_{i}$, we say that $x$ is greater than $y$ in the Lorenz ordering $\left(x \succsim_{L} y\right)$ if $\sum_{i=1}^{k} x_{i} \geq \sum_{i=1}^{k} y_{i}$, for all $k=1, \ldots, n-1$, with at least one strict inequality. This criterion induces a partial ordering on allocations which reflects their relative spread. When $x$ is greater than $y$ in the Lorenz ordering, the distribution $x$ is unambiguously "more egalitarian" than the distribution $y$. It is well known that this property is equivalent to saying that $y$ can be obtained from $x$ by means of a finite sequence of transfers "from the richer to the poorer". Moreover, the value of any symmetric relative index satisfying the Pigou-Dalton transfer principle and the Dalton population principle is higher at $y$ than at $x$ (see e.g., Atkinson (1970), Dasgupta, Sen and Starret (1973), Rostchild and Stiglitz (1973), Chakravarty (1999)). Before appealing to the Lorenz ordering, we present two minimal (and natural) requirements that are expected from a fair allocation.

First, a requirement of impartiality that says that symmetric agents should be treated alike.

Symmetry. $\gamma_{i}=\gamma_{n-i+1}$ for all $i=1, \ldots,\left[\frac{n}{2}\right]$.

Then, a principle that advocates a positive but limited discrimination towards users with less extreme locations in the model.

Partial Equalization. For all $i, j$ adjacent agents in $N$, let $\pi_{i j}=$ $\gamma_{i}-\gamma_{j}$. Then, if $j$ is closer than $i$ to the median agent of $N$, then $0 \leq \pi_{i j} \leq d$.

The concept of partial equalization, introduced by Le Breton and Weber (2003), says that, even though agents closer to the location of the facility might contribute less (given that the transportation cost they incur is lower), they cannot enjoy a reduction in their contribution higher than the distance 
with respect to agents farther away from the location of the facility. ${ }^{13}$

Let us now investigate the first approach to provide egalitarian allocations. We focus here on the nucleolus and the Shapley value for this context. ${ }^{14}$ It follows from Proposition 2 that the nucleolus is symmetric and satisfies the principle of partial equalization in our setting. As for the Shapley value, it turns out that, although it does not always provide secession-proof allocations, it can also be defended on the grounds of partial equalization. For instance, in the three-agent case, it is not difficult to see that the difference between the cost of the median agent and the corner agents is $\frac{d}{2}$ for the Shapley value and $\min \left\{\frac{f-d}{2}, d\right\}$ for the nucleolus. Thus,

$$
\begin{array}{ll}
\pi_{i j}(N) \leq \pi_{i j}(S h) & \text { if and only if } d \leq f \leq 2 d \\
\pi_{i j}(N) \geq \pi_{i j}(S h) & \text { if and only if } f>2 d
\end{array}
$$

Then we have

$$
\begin{array}{ll}
N \succsim_{L} S h & \text { if and only if } d \leq f \leq 2 d \\
S h \succsim_{L} N & \text { if and only if } f \geq 2 d
\end{array}
$$

Something similar happens for the case in which $n=4$. The Shapley value is then given by

$$
\left(\frac{f+5 d}{4}, \frac{3 f+7 d}{12}, \frac{3 f+7 d}{12}, \frac{f+5 d}{4}\right)
$$

From here, it follows that if $2 d \leq f \leq 3 d$, the environment would be $N$ efficient and the Shapley value for that environment prone to secession. It is also straightforward to see that $\pi_{i j}(S h)=\frac{2 d}{3}$ and $\pi_{i j}(N)=\min \left\{\frac{f-d}{2}, d\right\}$. Thus, $\pi_{i j}(N) \geq \pi_{i j}(S h)$ in all cases. However, we have that

$$
\begin{array}{ll}
N \succsim_{L} S h & \text { if } d \leq f \leq \frac{7}{3} d \\
S h \succsim_{L} N & \text { if } f \geq \frac{7}{3} d
\end{array}
$$

Finally, we now consider the second route to provide egalitarian allocations. Rather than focusing on equalizing the surplus of the coalitions, as the nucleolus does, we focus on equalizing the payments. To do that, a natural candidate is the allocation $E$ :

Equal-cost rule (E). For all $i \in N, E_{i}=\frac{c(N)}{n}$.

In general, $E$ is not a secession-proof rule, unless $f$ is sufficiently large. More precisely,

\footnotetext{
${ }^{13}$ The part of the requirement that says that an agent cannot contribute less than another agent closer to the median agent is usually referred to as monotonicity.

${ }^{14}$ Some insights on the Dutta-Ray solution and related solutions are also presented in Appendix A2.
} 
Proposition 5: The following statements hold:

(i) If $n$ is even then $E$ is a secession-proof rule if and only if $f \geq \frac{n^{2}}{4} d$

(ii) If $n$ is odd then $E$ is a secession-proof rule if and only if $f \geq \frac{(n-1)^{2}}{4} d$

In order to prove statement $(i)$, we have to verify the following inequalities:

$$
k \frac{c(N)}{n} \leq c(\{1, \ldots, k\}), \text { for all } k=1, \ldots, n,
$$

i.e.,

$$
\begin{array}{ll}
k\left(f+\frac{n^{2}}{4} d\right) \leq n\left(f+\frac{k^{2}}{4} d\right) & \text { for all } k \text { even between } 1 \text { and } n \\
k\left(f+\frac{n^{2}}{4} d\right) \leq n\left(f+\frac{k^{2}-1}{4} d\right) & \text { for all } k \text { odd between } 1 \text { and } n
\end{array}
$$

Note that any other coalition of $k$ agents would keep the left hand side of the inequality equal, but would increase the right hand side, making the resulting inequality less informative. Now, the above inequalities are equivalent to

$$
\begin{array}{ll}
f \geq \frac{k n}{4} d & \text { for all } k \text { even between } 1 \text { and } n \\
f \geq \frac{n(k(n-k)+1)}{4(n-k)} d & \text { for all } k \text { odd between } 1 \text { and } n
\end{array} .
$$

Thus, the maxima thresholds are for $k=n-2$ and $k=n-1$ respectively, i.e.,

$$
\frac{f}{d} \geq \max \left\{\frac{n(n-2)}{4}, \frac{n^{2}}{4}\right\}
$$

Therefore, $f \geq \frac{n^{2}}{4} d$, as desired.

Assume now that $n$ is odd. Then, the inequalities we have to verify become

$$
\begin{array}{ll}
k\left(f+\frac{n^{2}-1}{4} d\right) \leq n\left(f+\frac{k^{2}}{4} d\right) & \text { for all } k \text { even between } 1 \text { and } n \\
k\left(f+\frac{n^{2}-1}{4} d\right) \leq n\left(f+\frac{k^{2}-1}{4} d\right) & \text { for all } k \text { odd between } 1 \text { and } n
\end{array}
$$

Equivalently,

$$
\begin{array}{ll}
f \geq \frac{k}{4}\left(n-\frac{1}{n-k}\right) d & \text { for all } k \text { even between } 1 \text { and } n \\
f \geq \frac{k n+1}{4} d & \text { for all } k \text { odd between } 1 \text { and } n
\end{array} .
$$


Thus, the maxima thresholds are for $k=n-1$ and $k=n-2$ respectively, i.e.,

$$
\frac{f}{d} \geq \max \left\{\frac{(n-1)^{2}}{4}, \frac{(n-1)^{2}}{4}\right\} .
$$

Therefore, $f \geq \frac{(n-1)^{2}}{4} d$, as desired.

The final proposition of this section asserts that partial equalization is a necessary requirement for secession-proofness. More precisely, we show that if there exists a secession-proof allocation rule that violates partial equalization, then we can always obtain another secession-proof allocation that dominates the former one in the sense of Lorenz. Such fairness implication of secession-proofness was already pointed out by Le Breton and Weber (2003) in a continuous version of this one-dimensional model.

Proposition 6: If a secession-proof allocation violates partial equalization, then there exists a positive transfer of costs between two immediate neighbors such that the resulting allocation is also secession-proof.

Proof: Let $\gamma$ be a secession-proof allocation that violates partial equalization. Then, there exist two adjacent agents $i, j$, with $j$ is closer to the median agent than $i$, such that $\gamma_{i}-\gamma_{j}>d$. Let $\varepsilon$ be an arbitrarily small (positive) number and let $\gamma^{\varepsilon}$ denote the resulting allocation from $\gamma$ after transferring $\varepsilon$ from the cost of $i$ to the cost of $j$. Formally, $\gamma_{i}^{\varepsilon}=\gamma_{i}-\varepsilon$, $\gamma_{j}^{\varepsilon}=\gamma_{j}+\varepsilon$ and $\gamma_{k}^{\varepsilon}=\gamma_{k}$, for all $k \neq i, j$. We show next that $\gamma^{\varepsilon}$ is secessionproof.

If, on the contrary, $\gamma^{\varepsilon}$ is prone to secession, then there exists a coalition $S \subset N$ for which $\gamma^{\varepsilon}(S)>c(S) \geq \gamma(S)$. If so, $S$ must include agent $j$ and cannot include agent $i$. Thus, $\gamma^{\varepsilon}(S)-\gamma(S)=\varepsilon$. As $\varepsilon$ is arbitrarily small, this imposes that $c(S)=\gamma(S)$.

Let $\hat{S}$ be the resulting coalition from $S$ after replacing $j$ by $i$, i,e., $\hat{S}=$ $(S \backslash\{j\}) \cup\{i\}$. Then,

$$
\gamma(\hat{S})=\gamma(S)-\gamma_{j}+\gamma_{i}>\gamma(S)+d=c(S)+d .
$$

On the other hand, it is straightforward to show that $c(\hat{S}) \leq c(S)+d$. Thus, it follows from (15) that $\gamma(\hat{S})>c(\hat{S})$, which contradicts the fact that $\gamma$ is a secession-proof allocation. 


\section{Conclusions}

There are several natural questions that remain open in this line of research. With respect to public good projects, we could consider settings where public projects differ according to the needs that they are supposed to fulfill. ${ }^{15} \mathrm{An}$ individual could be a member of several communities simply because these communities provide different services. In such a setting, we could consider a set of public projects defined by two components: one that relates to the "physical" nature of the project (hospital, library, swimming pool) whereas the second captures characteristics of the project, such as its size or location. In such setting, it seems reasonable to assume that each player may consume each of the different "physical" public goods but may partner with other individuals who may exhibit different preferences over specific characteristics of the project. Under separability, it could be the case that even if each of the physical public goods is indivisible, a stable partition exists for each of them separately under full transferability (e.g., if the preferences are single peaked). We could consider public projects that are subject to congestion and capacity constraints, the topic that has been analyzed in operations research as the Capacited Facility Location Problem (CFLP), or to introduce a more general form of cost functions (Hajiaghayi, Madhian and Mirrokni (2003)). Finally, we could also examine the cases where individual demands for public projects are responsive to prices.

Many of the questions examined in this paper are intimately related to the growing literature on algorithmic game theory and mechanism design for combinatorial problems. This research conducted mostly by computer scientists and scholars in operations research focuses on approximation and algorithms as well as design of mechanisms for sharing the aggregate cost associated with the specific problem at hand. Most of the combinatorial optimization problems dealing with the computation of $C(S)$ are NP-hard. This implies that if algorithmic constraints are imposed on any coalition (including the grand coalition), $C(S)$ should be replaced by $\widehat{C}(S)>C(S)$. There do not exist cost sharing mechanisms which are simultaneously efficient, group-strategy proof and budget-balanced (e.g., Green et al, 1976). Moulin (1999) characterizes a whole class of budget-balanced and group-strategy proof mechanisms and, based on this result, Moulin and Shenker (2001) single out the mechanism associated with the Shapley value formula because it generates the smallest potential deviation from efficiency. Several authors have taken the alternative (more usual) route and have investigated the class

\footnotetext{
${ }^{15}$ On this issue see Alesina, Angeloni and Etro (2005).
} 
of group-strategy-proof mechanisms that are almost budget-balanced (e.g., Clarke, 1971; Groves, 1973; and, more recently, Moulin, 2009). To recall, a cost sharing mechanism is a collection of cost sharing allocations $\phi^{S}$ for a coalition $S$ that assigns no cost to those outside of $S$. Given $\alpha \leq 1$, the mechanism is $\alpha$-budget balanced if:

$$
\alpha C(S) \leq \sum_{i \in S} \phi_{i}^{S} \leq C(S) \text { for all } S \subseteq N
$$

It is cross-monotone if $S \subseteq T$ implies $\phi_{i}^{S} \leq \phi_{i}^{T}$ for all $i \in S$. Moulin (1999) has shown that if a cost sharing mechanism is cross-monotone then one can construct a group strategy-proof mechanism to elicit the information on the willingness to pay for the service of the individual. The logical relationship with the question considered in our paper rests on the fact that for every $\alpha$-budget balanced cross-monotone cost-sharing mechanism the inequality $1+\varepsilon_{m}^{*} \leq \frac{1}{\alpha}$ holds. Any information on values of $\alpha$ (called in this literature, the budget balance factor) for which there exists a $\alpha$-budget balanced cross monotonic cost sharing mechanism yields an upper bound on $1+\varepsilon_{m}^{*}$. For the metric facility location problem considered in this paper, Pal and Tardos (2003) exhibited a $\frac{1}{3}$-budget balanced cross-monotonic cost sharing mechanism. Immorlica, Mahdian and Mirrokni (2008) determine upper bounds on $\alpha$ for several cost sharing problems. Devanur, Mihail and Vazirani (2005) demonstrate the existence of a 0.537-budget balanced, strategy-proof (which is weaker that cross-monotonicity) and belong to the modified version of the core. Many of combinatorial games can be expressed as linear programs with integer constraints. As emphasized by Jain and Varizani (2001), up to some qualifications, the best budget-balance factor is bounded by the integrality gap of the natural LP-relaxation of the problem.

\section{Appendix}

Some of the notions used in the paper as well as the statement and (or) the proofs of some of the results are stated in the following three appendices.

\section{Appendix A1. Least Core(s) and Nucleolus}

Consider a cooperative game with transferable utility as the one introduced in subsection 3.1. It is called super-additive if $V(S \cup T) \geq V(S)+$ $V(T)$ for all $S, T \subseteq N$ such that $S \cap T=\varnothing$. A player $i \in N$ is a nullplayer (dummy) of $(N, V)$ if $V(S \cup\{i\})=V(S)(V(S \cup\{i\})=V(S)+$ 
$V\{i\})$. Hereafter, we denote by $X_{P O} \equiv\left\{y \in \mathbb{R}^{n} \mid \sum_{i=1}^{n} y^{i}=V(N)\right\}$ the set of (pre)imputations (or Pareto optimal imputations) and by $X_{I R} \equiv$ $\left\{y \in \mathbb{R}^{n} \mid \sum_{i=1}^{n} y^{i}=V(N), y^{j} \geq V(\{j\}) \forall j \in N\right\}$ the set of imputations i.e., the set of individually rational preimputations.

Let $X$ be a compact and convex subset of $\mathbb{R}^{n}$ and let $x \in X$. We denote by $\theta(x)$ the $2^{n}$-dimensional vector ${ }^{16}$ whose components are the numbers $e(S, x) \equiv V(S)-\sum_{i \in S} x^{i}$ for $\emptyset \subseteq S \subseteq N$ arranged according to their magnitude, i.e., $\theta^{i}(x) \geq \theta^{j}(x)$ for $1 \leq i \leq j \leq 2^{n}$. The nucleolus of $(N, V)$ with respect to $X$ is the unique ${ }^{17}$ vector $x^{*}=N_{u}(N, V) \in X$ such that $\theta\left(x^{*}\right)$ is minimal, in the sense of the lexicographic order, of the sets $\{\theta(y) \mid y \in X\}$. The nucleolus of $(N, V)$ with respect to $X_{I R}$ will be called hereafter the nucleolus as originally defined by Schmeidler (1969). ${ }^{18}$ We denote by $\psi(x)$ the $2^{2 n}$-dimensional vector whose components are the numbers $e(S, x)-$ $e(T, x)$ for $\emptyset \subseteq S, T \subseteq N$ arranged in the descending order, i.e., $\psi^{i}(x) \geq$ $\psi^{j}(x)$ for $1 \leq i \leq j \leq 2^{2 n}$.

Given a real number $\epsilon$, the $\epsilon$-core of $(N, V)$ is the set $C_{\epsilon} \equiv\left\{x \in X_{P O}: e(S, x) \leq \epsilon\right.$ for all $\left.\varnothing \nsubseteq S \varsubsetneqq N\right\}$. The least core ${ }^{19}$ of $(N, V)$, denoted $L C(V, N)$ is the intersection of all nonempty $\epsilon$ - core of $(N, V) .{ }^{20}$ If $(N, V)$ is super-additive, then $L C(V, N) \subseteq X_{I R}$. In such case, $L C(V, N)$ consists of the vectors $x$ such that $\theta_{1}(x)=\theta_{1}\left(x^{*}\right)$. Note that then, $x^{*} \in$ $L C(V, N)$.

Given a TU cooperative game $V$ over the set of players $N$, the least core $^{21}$ of $V$ is, by definition, the set $L C(V)$ of allocations $x$ that are optimal

\footnotetext{
${ }^{16}$ This vector is called the vector of excesses attached to $x$.

${ }^{17}$ For a proof of uniqueness, see Peleg and Sudhölter (2003).

${ }^{18} \mathrm{In}$ contrast, the prenucleolus is the nucleolus with respect to $X \equiv$ $\left\{y \in \mathbb{R}^{n} \mid \sum_{i=1}^{n} y^{i}=V(N)\right\}$. If the cooperative game is zero-monotonic, i.e., if $V(S \cup$ $\{i\})-V(S) \geq V(\{i\})$ for all $i \in N$ and $S \subseteq N \backslash\{i\}$, the difference between the prenucleolus and the nucleolus disappears.

${ }^{19}$ The notion of least core was first introduced by Maschler, Peleg and Shapley (1979). Each payoff vector of the least core of a zero-monotonic game is individually rational.

${ }^{20}$ In recent years, the least core has received increasing attention from scholars in the area of combinatorial cooperative games. A combinatorial cooperative game is a cooperative game where the value $V(S)$ or cost $C(S)$ of coalition $S$ arises as the optimal value of a combinatorial maximization or minimization problem defined by $S$. Among the most popular (in addition to the facility location problem), we can cite: network flow games, minimum-cost spanning tree games (e.g., Granot and Huberman, 1981; 1984), Steiner tree games, bin packing games, traveling salesman games, scheduling games and, naturally, matching games.

${ }^{21}$ A very similar concept is considered by Montero (2006) under the name nucleus. Both concepts coincide if the game is super-additive and the core is empty. If the core is nonempty, the nucleus coincides with the core.
} 
solutions to the linear program:

$$
\varepsilon^{*}=\operatorname{Min} \varepsilon
$$

such that $x(N)=V(N)$ and $x(S) \geq V(S)-\varepsilon$ for all $S \subseteq N, S \neq N, \varnothing$.

The optimal value $\varepsilon^{*}$ is the least core value of the game $V$ :

$$
\varepsilon^{*}=\operatorname{Min}_{x: x(N)=V(N) S \subseteq N, S \neq N, \varnothing}^{\operatorname{Max}} e(x, S)
$$

In the case of a TU cost-sharing cooperative game $C$ over the set of players $N$, the definition of $L C(C)$ and $\varepsilon^{*}$ is similar:

$\varepsilon^{*}=\operatorname{Min} \varepsilon$ such that $x(N)=C(N)$ and $x(S) \leq C(S)+\varepsilon$ for all $S \subseteq N, S \neq N, \varnothing$.

Several variants of the least core and the least core value have been discussed in the literature. We may consider more general valuation of the excesses. Given an arbitrary penalty function $f: 2^{N} \rightarrow \mathbb{R}$, the $f$-Least Core of $V$, $L C_{f}(V)$ offers a way yo address this issue (e.g., Faigle, Kern and Paulusma, 2000). ${ }^{22}$ It is the set of allocations that are optimal solutions to the linear program

$$
\varepsilon_{f}^{*}=\operatorname{Min} \varepsilon
$$

such that $x(N)=V(N)$, and $x(S) \geq V(S)-\varepsilon f(S)$ for all $S \subseteq N, S \neq N, \varnothing$,

and, similarly, in the cost-sharing game.

When $f(S)=1$ for all $S \subseteq N$, we obtain the (standard, sometimes called additive) least core defined before. When $f(S)=\# S$, it is so-called percapita least cost (Grotte (1970), Wallmeier (1983)). Finally, when $f(S)=$ $V(S)$, or $f(S)=C(S)$ in the cost-sharing version of the game, we have the (multiplicative) least core $L C_{m}(V)$ (Faigle and Kern (1993)). This is the version of the least core considered in our paper. The multiplicative version of the nucleolus has been introduced and analyzed by Faigle et al. (1998), who called it nucleon.

It is useful for alternative interpretations to consider the multiplicative least core as describing a model of taxation where coalitions are taxed proportionally to their value. ${ }^{23}$ Cooperative games with a non-empty core under taxation at rate $\varepsilon$ are $\varepsilon$-balanced. Note that if we define the allocation $y$ as $\frac{x}{1+\varepsilon}$ in the case of cost-sharing games, we have

$$
y(N)=\frac{C(N)}{1+\varepsilon} \text { and } y(S) \leq C(S) \text { for all } S \subseteq N, S \neq N, \varnothing .
$$

\footnotetext{
${ }^{22}$ See Kern and Paulusma (2009) for an application to flow games.

${ }^{23}$ This idea has appeared first in Driessen and Tijs (1986).
} 
Of course, if the core of $C$ is empty, $\varepsilon_{m}^{*}>0$ and $y(N)<C(N)$. Instead of the taxation model, we have a subsidy setting: which fraction of the total cost should be subsidized by an external source to satisfy the standalone constraints of the proper coalitions? The problem of determining the multiplicative least core $L C_{m}(V)$ and the multiplicative least core value $\varepsilon_{m}^{*}$ amounts to solve the linear program:

$$
\operatorname{Max} x(N)
$$

such that $x(N)=C(N)$ with $x(S) \leq C(S)$ for all $S \subseteq N$.

We have:

$$
1+\varepsilon_{m}^{*}=\frac{C(N)}{x^{*}(N)} \text { with } 1+\varepsilon_{m}^{*}=\underset{x: x(N)=V(N) S \subseteq N, S \neq N, \varnothing}{\operatorname{Min}} \frac{x(S)}{C(S)} .
$$

The formula is the same as in the additive case except that here the excesses are expressed in a multiplicative form. From a practical perspective, it is of primary importance to be able to compute the (multiplicative) least cost value $\varepsilon_{m}^{*}$. To some extent this value indicates the distance from having a nonempty core. Faigle and Kern (1993) establish sharp bounds on $\varepsilon_{m}^{*}$ in matching games on (not necessarily bipartite) graphs. They also derive upper and lower bounds on $\varepsilon_{m}^{*}$ and show that $\varepsilon_{m}^{*} \leq \frac{1}{2}$ in bin packing games. They also argue that for Euclidean random traveling salesman games $\varepsilon_{m}^{*} \simeq$ 0.06, with high probability. For a subclass of bin packing games, Kuipers (1998) has shown that $\varepsilon_{m}^{*}=\frac{1}{7}$. Woeginger (1995) proves that for any bin packing game $\varepsilon_{m}^{*} \leq \frac{1}{3}$. Faigle and Kern (1998) improve their earlier results on $\varepsilon_{m}^{*}$ in bin packing games. Blaser and Shankar Ram (2008) present bounds on $\varepsilon_{m}^{*}$ for the class of metric salesman games. In a continuous bidimensional Euclidean version of the facility location problem, Drèze et al. (2008) proved that $\varepsilon_{m}^{*} \simeq 0.0019$ !

\section{Appendix A2. Dutta-Ray and other Egalitarian Solutions}

Dutta and Ray (1989) (DR - hereafter) define a solution concept (that they call the egalitarian solution) for transferable utility cooperative games in characteristic function form. In that framework individuals believe in equality as a desirable social goal, although private preferences dictate selfish behavior. This latter aspect implies that the solution outcome(s) must satisfy core-like participation constraints while the concern for equality entails a choice of Lorenz maximal elements from within the set of payoffs satisfying the participation constraints. Despite the Lorenz domination relation being 
a partial ranking, they show that the egalitarian solution is unique whenever it exists. In order to develop the DR egalitarian allocation we need first a piece of notation. The Lorenz map $E$ is defined on the domain of vectors with the same dimension and mean. For each such set $A, E(A)$ is the set of all allocations in $A$ that are not Lorenz dominated within $A$. Formally,

$E(A)=\left\{x \in A: \nexists y \in A\right.$ such that $\sum_{i=1}^{k} x_{i} \leq \sum_{i=1}^{k} y_{i}$, for all $\left.k=1, \ldots, n-1,\right\}$

where $x, y \in \mathbb{R}^{n}$ are such that $x_{1} \leq x_{2} \leq \ldots \leq x_{n}, y_{1} \leq y_{2} \leq \ldots \leq y_{n}$, and $\sum_{i=1}^{n} x_{i}=\sum_{i=1}^{n} y_{i}$, and one of the above inequalities is strict for some $k$. Note, first, that $E(A)$ may be empty, but that $E(A)$ is nonempty whenever $A$ is closed. Second, for $A \subset \mathbb{R}, E(A)=A$. Next, the Lorenz cores of coalitions are defined recursively.

- The Lorenz core of a singleton coalition is $L(\{i\})=V(\{i\})$.

- Now suppose that the Lorenz cores for all coalitions of cardinality $k$ or less have been defined, where $1<k<n$. The Lorenz core of a coalition $S$ of size $(k+1)$ is defined by

$L(S)=\left\{x \in \mathbb{R}^{k+1}: \sum_{i=1}^{k+1} x_{i}=V(S)\right.$ and $\nexists T \subset S, y \in E(L(T))$ such that $\left.y>x(T),\right\}$

where $x(T)$ denotes the projection of $x$ over the coalition $T$.

If $x \in S$, and there is $T \subset S, y \in E(L(T))$ such that $y>x(T)$, then we say that $y$ Lorenz-blocks (L-blocks) $x$. We shall also say in this case that $T$ L-blocks $x$. A coalition $S$ will be called viable if $E L(S)$ is nonempty. An egalitarian allocation exists if the grand coalition is viable. $E L(N)$ will denote the set of egalitarian allocations, that as DR show will be a singleton set. It is straightforward to show that $C(S) \subseteq L(S)$, where $C(S)$ denotes the core of coalition $S$.

DR show that if the game $(\mathrm{N}, \mathrm{V})$ is convex the egalitarian solution exists, belongs to the core and Lorenz dominates every other allocation in the core. These assertions are not true in general: the core can be nonempty but the egalitarian allocation may fail to exist; the egalitarian allocation exists but the core is empty or the core is nonempty and the egalitarian allocation exists, but the egalitarian allocation does not lie in the core.

Prompted by the conflict between the core and DR's egalitarian solution in general (not necessarily convex) games, Hougaard, Thorlund-Petersen and 
Peleg (2001) propose to focus on the so-called Lorenz maximal imputations, which is defined as the subset of the core consisting of the allocations that are not Lorenz dominated by any other allocation of the core. ${ }^{24}$ As they acknowledge, the problem with this solution concept is that, typically, it is not single valued. In order to bypass this problem, Arin and Iñarra (2001) propose, another solution concept, the leximin stable allocation, which is derived from the application of the Rawlsian criterion on the core. Hougaard et al. (2001) define the Lorenz maximal imputations as the set

$$
L M(N, v)=E(C(N, V)),
$$

where $C(N, v)$ denotes the core of the game $(N, v)$.

Let $x, y \in \mathbb{R}^{n}$ such that $x_{1} \leq x_{2} \leq \ldots \leq x_{n}, y_{1} \leq y_{2} \leq \ldots \leq y_{n}$. We say that $y$ leximin dominates $x\left(x \succ_{l} y\right)$ if there is some $k \in\{0,1,2, \ldots, n-1\}$ such that $x_{i}=y_{i}$ for $i=1, \ldots, k$ and $x_{k+1}>y_{k+1}$. Then, the leximin stable allocation is defined as

$$
L X(N, V)=x \in C(N, V) \text { such that } x \succ_{l} y \text {, for all } y \in C(N, V) \backslash\{x\} .
$$

We now describe the main features of these egalitarian solutions in the context of our cost-sharing game $(N, C)$ (normalized to obtain a surplussharing game $(N, V))$ in the cases where $n=3,4$ and 5 .

Example 1: Consider first the three-agent case. Then, $N=\{1,2,3\}$, $v(1)=v(2)=v(3)=0, v(12)=v(23)=f-d ; v(13)=f-2 d ;$ $v(N)=2 f-2 d$. Then,

- $L(i)=E(L(i))=0$;

- $E(L(23))=E(L(12))=\left\{\begin{array}{ll}\emptyset & \text { if } f<d \\ \left(\frac{f-d}{2}, \frac{f-d}{2}\right) & \text { if } f \geq d\end{array} ; E(L(13))=\right.$ $\left\{\begin{array}{ll}\emptyset & \text { if } f<2 d \\ \left(\frac{f-2 d}{2}, \frac{f-2 d}{2}\right) & \text { if } f \geq 2 d\end{array}\right.$.

- $L(N)=\left\{\left(x_{1}, x_{2}, x_{3}\right) \in \mathbb{R}_{+}^{3}: \sum x_{i}=2 f-2 d ;\right.$ For $g \geq d,\left(\frac{f-d}{2}, \frac{f-d}{2}\right) \ngtr$ $\left(x_{1}, x_{2}\right),\left(x_{2}, x_{3}\right)$; For $\left.f \geq 2 d,\left(\frac{f-2 d}{2}, \frac{f-2 d}{2}\right) \ngtr\left(x_{1}, x_{3}\right)\right\}$;

- $E(L(N))= \begin{cases}\emptyset & \text { if } f<d \\ \left(\frac{2}{3}(f-d), \frac{2}{3}(f-d), \frac{2}{3}(f-d)\right) & \text { if } f \geq d\end{cases}$

Thus, the DR egalitarian solution is precisely the equal-split solution, provided $f \geq d$. If we move back from $(\mathrm{N}, \mathrm{V})$ to the cost-sharing

\footnotetext{
${ }^{24}$ Arin and Iñarra (2001) refer to this solution as the Lorenz stable set.
} 
game, we would have that the solution is $\left(\frac{f+2 d}{3}, \frac{f+2 d}{3}, \frac{f+2 d}{3}\right)$. It follows from Proposition 3 that this allocation is secession-proof and hence the other two solutions proposed above provide here the same solution, as expected.

Example 2: We now consider the four-agent case. Then, $N=\{1,2,3,4\}$, $v(1)=v(2)=v(3)=v(4)=0, v(12)=v(23)=v(34)=f-d$; $v(13)=v(24)=f-2 d ; v(14)=f-3 d ; v(123)=v(234)=2 f-2 d$; $v(134)=v(124)=2 f-3 d ; v(N)=3 f-4 d$. Then,

- $L(i)=E(L(i))=0$;

$E(L(34))=E(L(23))=E(L(12))=\left\{\begin{array}{ll}\emptyset & \text { if } f<d \\ \left(\frac{f-d}{2}, \frac{f-d}{2}\right) & \text { if } f \geq d\end{array} ;\right.$
$E(L(13))=E(L(24))=\left\{\begin{array}{ll}\emptyset & \text { if } f<2 d \\ \left(\frac{f-2 d}{2}, \frac{f-2 d}{2}\right) & \text { if } f \geq 2 d\end{array} ; E(L(14))=\right.$ $\left\{\begin{array}{ll}\emptyset & \text { if } f<2 d \\ \left(\frac{f-3 d}{2}, \frac{f-3 d}{2}\right) & \text { if } f \geq 3 d\end{array}\right.$.

- $E L(123)=E L(234)= \begin{cases}\emptyset & \text { if } f<d \\ \left(\frac{2}{3}(f-d), \frac{2}{3}(f-d), \frac{2}{3}(f-d)\right) & \text { if } f \geq d\end{cases}$ $E L(124)=E L(134)= \begin{cases}\emptyset & \text { if } f<2 d \\ \left(\frac{f-d}{2}, f-2 d, \frac{f-d}{2}\right) & \text { if } 2 d \leq f \leq 3 d \\ \left(\frac{2 f-3 d}{3}, \frac{2 f-3 d}{3}, \frac{2 f-3 d}{3}\right) & \text { if } f>3 d\end{cases}$

- $L(N)=\left\{\left(x_{1}, x_{2}, x_{3}, x_{4}\right): \sum x_{i}=3 f-4 d\right.$; For $f \geq d,\left(\frac{f-d}{2}, \frac{f-d}{2}\right) \ngtr$ $\left(x_{1}, x_{2}\right),\left(x_{2}, x_{3}\right),\left(x_{3}, x_{4}\right)$; For $f \geq 2 d,\left(\frac{f-2 d}{2}, \frac{f-2 d}{2}\right) \ngtr\left(x_{1}, x_{3}\right),\left(x_{2}, x_{4}\right)$; For $f \geq 3 d,\left(\frac{f-3 d}{2}, \frac{f-3 d}{2}\right) \ngtr\left(x_{1}, x_{4}\right)$; For $f \geq d,\left(\frac{2}{3}(f-d), \frac{2}{3}(f-\right.$ d), $\left.\frac{2}{3}(f-d)\right) \ngtr\left(x_{1}, x_{2}, x_{3}\right),\left(x_{2}, x_{3}, x_{4}\right)$; For $2 d \leq f \leq 3 d,\left(\frac{f-d}{2}, f-\right.$ $\left.2 d, \frac{f-d}{2}\right) \ngtr\left(x_{1}, x_{2}, x_{4}\right),\left(x_{1}, x_{3}, x_{4}\right)$; For $f>3 d,\left(\frac{2 f-3 d}{3}, \frac{2 f-3 d}{3}, \frac{2 f-3 d}{3}\right) \ngtr$ $\left.\left(x_{1}, x_{2}, x_{4}\right),\left(x_{1}, x_{3}, x_{4}\right)\right\}$

- $E(L(N))= \begin{cases}\emptyset & \text { if } f<2 d \\ \left(f-2 d, \frac{f}{2}, \frac{f}{2}, f-2 d\right) & \text { if } 2 d \leq f \leq 4 d \\ \left(\frac{3 f-4 d}{4}, \frac{3 f-4 d}{4}, \frac{3 f-4 d}{4}, \frac{3 f-4 d}{4}\right) & \text { if } f>4 d\end{cases}$

Thus, the DR egalitarian solution is precisely the equal-split solution, for high values of the facility cost. More precisely, for $f \geq 4 d$. If we move back from $(\mathrm{N}, \mathrm{V})$ to the cost-sharing game, we would have that the solution is $\left(\frac{f+4 d}{4}, \frac{f+4 d}{4}, \frac{f+4 d}{4}, \frac{f+4 d}{4}\right)$. By Proposition 3 , this solution is secession proof. Thus, under the condition, $f \geq 4 d$ the 
other two solutions proposed above provide here the same solution, as expected. If $2 d \leq f \leq 4 d$, then the Lorenz maximal imputations is just a singleton set: $\left\{\left(2 d, \frac{f}{2}, \frac{f}{2}, 2 d\right)\right\}$ and therefore the leximin stable solution for this case is also $\left(2 d, \frac{g}{2}, \frac{f}{2}, 2 d\right)$. The corresponding solution to the associated TU-fame would be $\left(f-2 d, \frac{f}{2}, \frac{f}{2}, f-2 d\right)$, the DR egalitarian solution.

\section{Appendix A3. The Nucleolus in the One-dimensional Metric Environment}

In this appendix, we explicitly derive the nucleolus in the one dimensional equidistant environment with no more than seven players. For the specific case of three agents, we examine a more general case in which the two individuals at the extreme are not necessarily equidistant from the median.

\section{The three-agent case}

let $N=\{1,2,3\}$. Let $x$ denote the distance between 1 and 2 and $y$ the distance between 2 and 3 , and assume, without loss of generality, that $x \leq y$.

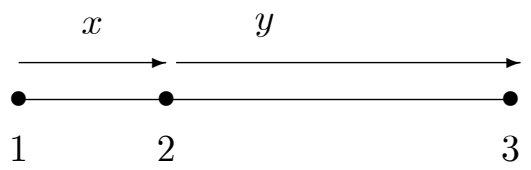

The next lemmata conveys our main results for this case.

Lemma 1: $N$-Efficiency holds if and only if $f \geq y$.

If $N$-efficiency holds, then

$$
c(N)=f+x+y \leq c(\{1,2\}+c(\{3\})=2 f+x,
$$

or, equivalently, $f \geq y$. As for the converse implication, it suffices to note that $\{\{1,2\},\{3\}\}$ is the "most expensive" partition of $N$.

Lemma 2: The nucleolus is given by

$$
\left(\frac{f+x}{2}, \frac{x+y}{2}, \frac{f+y}{2}\right) \text { if } y \leq f \leq 2 x+y
$$




$$
\begin{gathered}
\left(\frac{f+4 x+y}{4}, \frac{f+y}{4}, \frac{f+y}{2}\right) \text { if } 2 x+y \leq f \leq 3 y \\
\left(\frac{f}{3}+x, \frac{f}{3}, \frac{f}{3}+y\right) \text { if } f \geq 3 y
\end{gathered}
$$

In order to prove this lemma, assume first that $y \leq f \leq 2 x+y$. Let

$$
\gamma^{*}=\left(\frac{f+x}{2}, \frac{x+y}{2}, \frac{f+y}{2}\right)
$$

It is straightforward to show that $\sum_{i=1}^{n} \gamma_{i}^{*}=f+x+y=c(N)$. Note that the distribution of surpluses (cost-savings) associated to $\gamma^{*}$ is given by

$$
\begin{gathered}
e\left(\gamma^{*},\{1\}\right)=e\left(\gamma^{*},\{2,3\}\right)=\frac{f-x}{2}, \\
e\left(\gamma^{*},\{3\}\right)=e\left(\gamma^{*},\{1,2\}\right)=\frac{f-y}{2}, \\
e\left(\gamma^{*},\{2\}\right)=\frac{2 f-x-y}{2}, \\
e\left(\gamma^{*},\{13\}\right)=\frac{x+y}{2} .
\end{gathered}
$$

It is straightforward to demonstrate that, for the range being considered, $y \leq f \leq 2 x+y$,

$$
\min _{S} e\left(\gamma^{*}, S\right)=\min \left\{\frac{f-y}{2}, \frac{x+y}{2}\right\} \geq 0,
$$

which shows that $\gamma^{*}$ is secession-proof for such a range.

Let now $\gamma=\left(\gamma_{1}, \gamma_{2}, \gamma_{3}\right)$ be the secession-proof allocation that lexicographically maximizes the distribution of cost-savings in this case. Note that, if $y \leq f \leq 2 x+y$, then $\frac{f-y}{2} \leq \frac{f-x}{2} \leq \frac{x+y}{2}$ and, in particular,

$$
e\left(\gamma^{*}, S\right) \geq \frac{f-y}{2} \text { for all } S .
$$

Assume, by contradiction, that $\min \{e(\gamma, S)\}>\frac{f-y}{2}$. Then, in particular,

$$
\min \{e(\gamma,\{1,2\}), e(\gamma,\{3\})\}>\frac{f-y}{2},
$$


and, therefore,

$$
\gamma_{1}+\gamma_{2}<c(\{1,2\})-\frac{f-y}{2}=f+x-\frac{f-y}{2}
$$

and

$$
\gamma_{3}<c(\{3\})-\frac{f-y}{2}=f-\frac{f-y}{2}
$$

Adding (17) and (18) we have

$$
\sum_{i=1}^{3} \gamma_{i}<2 f+x-(f-y)=f+x+y
$$

However, by secession-proofness,

$$
\sum_{i=1}^{3} \gamma_{i}=c(N)=f+x+y,
$$

which represents a contradiction. Thus,

$$
e(\gamma,\{1,2\})=e(\gamma,\{3\})=\frac{f-y}{2},
$$

and, therefore,

$$
\gamma_{3}=f-\frac{f-y}{2}=\frac{f+y}{2}=\gamma_{3}^{*} .
$$

Given the distribution of cost-savings associated to $\gamma^{*}$, and the fact that the domain condition $y \leq f \leq 2 x+y$ for this case guarantees $\frac{f-y}{2} \leq \frac{f-x}{2} \leq \frac{x+y}{2}$, we know that

$$
\min \{e(\gamma,\{1\}), e(\gamma,\{2,3\})\} \geq \frac{f-x}{2} .
$$

If, by contradiction, $\min \{e(\gamma,\{1\}), e(\gamma,\{2,3\})\}>\frac{f-x}{2}$, then

$$
\gamma_{2}+\gamma_{3}<c(\{2,3\})-\frac{f-x}{2}=f+y-\frac{f-x}{2}
$$

and

$$
\gamma_{1}<c(\{1\})-\frac{f-x}{2}=f-\frac{f-x}{2} .
$$

Adding (20) and (21) we have

$$
\sum_{i=1}^{3} \gamma_{i}<2 f+y-(f-x)=f+x+y
$$


which, again, violates secession-proofness. Thus,

$$
e(\gamma,\{2,3\})=e(\gamma,\{1\})=\frac{f-x}{2},
$$

and, therefore,

$$
\gamma_{1}=f-\frac{f-x}{2}=\frac{f+x}{2}=\gamma_{1}^{*}
$$

Finally, by secession-proofness,

$$
\gamma_{2}=c(N)-\gamma_{1}-\gamma_{3}=\frac{y+x}{2}=\gamma_{2}^{*},
$$

as desired.

We now move to the case in which $2 x+y \leq f \leq 3 y$. Let

$$
\gamma^{*}=\left(\frac{f+4 x+y}{4}, \frac{f+y}{4}, \frac{f+y}{2}\right),
$$

Then, the distribution of cost-savings associated to $\gamma^{*}$ is given by

$$
\begin{gathered}
e\left(\gamma^{*},\{1,3\}\right)=e\left(\gamma^{*},\{2,3\}\right)=\frac{f+y}{4}, \\
e\left(\gamma^{*},\{3\}\right)=e\left(\gamma^{*},\{1,2\}\right)=\frac{f-y}{2}, \\
e\left(\gamma^{*},\{1\}\right)=\frac{3 f-4 x-y}{4}, e\left(\gamma^{*},\{2\}\right)=\frac{3 f-y}{4} .
\end{gathered}
$$

Let $\gamma=\left(\gamma_{1}, \gamma_{2}, \gamma_{3}\right)$ be the secession-proof allocation that lexicographically maximizes the distribution of cost-savings in this case. An analogous argument to the one in the previous case allows us to show here too that

$$
e(\gamma,\{1,2\})=e(\gamma,\{3\})=\frac{f-y}{2},
$$

and, therefore,

$$
\gamma_{3}=f-\frac{f-y}{2}=\frac{f+y}{2}=\gamma_{3}^{*} .
$$

Now, given the distribution of cost-savings associated to $\gamma^{*}$, and the fact that the domain condition $2 x+y \leq f \leq 3 y$ for this case guarantees that $\frac{f-y}{2} \leq \frac{f+y}{4} \leq \frac{3 f-4 x-y}{4}$, we know that $\min \{e(\gamma,\{1,3\}), e(\gamma,\{2,3\})\} \geq \frac{f+y}{4}$. Assume, by contradiction, that

$$
\min \{e(\gamma,\{1,3\}), e(\gamma,\{2,3\})\}>\frac{f+y}{4} .
$$


Then,

$$
\gamma_{2}+\gamma_{3}<c(\{2,3\})-\frac{f+y}{4}=\frac{3}{4}(f+y)
$$

and

$$
\gamma_{1}+\gamma_{3}<\frac{3}{4}(f+y)+x
$$

Adding (22) and (23) we have

$$
\gamma_{1}+\gamma_{2}+2 \gamma_{3}<\frac{3}{2}(f+y)+x
$$

Since $\gamma_{3}=\frac{f+y}{2}$ the above translates into

$$
\gamma_{1}+\gamma_{2}<\frac{f+y}{2}+x .
$$

which, again, represents a contradiction with secession-proofness, as $\gamma_{1}+$ $\gamma_{2}=f+x+y-\frac{f+y}{2}=\frac{f+y}{2}+x$. Thus,

$$
e(\gamma,\{2,3\})=e(\gamma,\{1,3\})=\frac{f+y}{4},
$$

and, therefore,

$\gamma_{1}=c(\{1,3\})-\frac{f+y}{4}-\gamma_{3}=f+x+y-\frac{f+y}{4}-\frac{f+y}{2}=\frac{f+4 x+y}{4}=\gamma_{1}^{*}$,

and

$$
\gamma_{2}=c(\{2,3\})-\frac{f+y}{4}-\gamma_{3}=f+y-\frac{f+y}{4}-\frac{f+y}{2}=\frac{f+y}{4}=\gamma_{2}^{*},
$$

as desired.

Finally, we focus on the case in which $f \geq 3 y$. Let

$$
\gamma^{*}=\left(\frac{f}{3}+x, \frac{f}{3}, \frac{f}{3}+y\right)
$$

Then, the distribution of cost-savings associated to $\gamma^{*}$ is given by

$$
\begin{gathered}
e\left(\gamma^{*},\{1,3\}\right)=e\left(\gamma^{*},\{1,2\}\right)=e\left(\gamma^{*},\{2,3\}\right)=\frac{f}{3} \\
e\left(\gamma^{*},\{1\}\right)=\frac{2 f}{3}-x, e\left(\gamma^{*},\{2\}\right)=\frac{2 f}{3}, e\left(\gamma^{*},\{3\}\right)=\frac{2 f}{3}-y .
\end{gathered}
$$


Let $\gamma=\left(\gamma_{1}, \gamma_{2}, \gamma_{3}\right)$ be the secession-proof allocation that lexicographically maximizes the distribution of cost-savings in this case. Given the distribution of cost-savings associated to $\gamma^{*}$, and the fact that the domain condition $f \geq 3 y$ for this case guarantees $\frac{2 f}{3}-y \geq \frac{f}{3}$, we know that $\min \{e(\gamma,\{1,3\}), e(\gamma,\{1,2\}), e(\gamma,\{2,3\})\} \geq \frac{f}{3}$.

Assume that $\min \{e(\gamma,\{1,3\}), e(\gamma,\{1,2\}), e(\gamma,\{2,3\})\}>\frac{f}{3}$. Then,

$f<c(\{1,2\})+c(\{1,3\})+c(\{2,3\})-2 c(N)=(f+x)+(f+x+y)+(f+y)-2(f+x+y)$,

a contradiction. Thus,

$$
e(\gamma,\{1,2\})=e(\gamma,\{2,3\})=e(\gamma,\{1,3\})=\frac{f}{3},
$$

and, therefore,

$$
\gamma_{i}+\gamma_{j}=c(\{i, j\})-\frac{f}{3},
$$

for all pair of agents $i$ and $j$. It is straightforward to conclude from here that

$$
\gamma=\left(\frac{f}{3}+x, \frac{f}{3}, \frac{f}{3}+y\right)=\gamma^{*}
$$

as desired.

This lemma shows us that there is a main difference worth remarking between the equidistant and general three-agent cases. In the general case we have just described, there is an intermediate case, that was not feasible in the equidistant case (if $x=y$, then $x+2 y=3 y$, and therefore only $3 y=3 x$ would emerge as a threshold separating both cases).

Corollary 1: For the equidistant case $(x=y=d)$, the nucleolus is given by

$$
\begin{gathered}
\left(\frac{f+d}{2}, d, \frac{f+d}{2}\right) \text { if } d \leq f \leq 3 d \\
\left(\frac{f}{3}+d, \frac{f}{3}, \frac{f}{3}+d\right) \text { if } f \geq 3 d
\end{gathered}
$$

\section{The four-agent case}

Let now $N=\{1,2,3,4\}$. We know from Proposition 3 that $N$-Efficiency holds in this setting if and only if $f \geq 2 d$. Then, we have the following: 
Lemma 3: The nucleolus is given by

$$
\left(\frac{f+6 d}{4}, \frac{f+2 d}{4}, \frac{f+2 d}{4}, \frac{f+6 d}{4}\right)
$$

For each $S \in 2^{N}$, let $e(\gamma, S)$ be the total amount that the members of coalition $S$ save as a group relative to their stand-alone cost, when $\gamma$ is implemented. Formally,

$$
e(\gamma, S)=c(S)-\gamma(S)=f+\left(\sum_{k=0}^{\left[\frac{s}{2}\right]-1}(\sigma(s-k)-\sigma(k+1))\right) \cdot d-\sum_{i \in S} \gamma_{i}
$$

Let $\gamma^{*}=\left(\frac{f+6 d}{4}, \frac{f+2 d}{4}, \frac{f+2 d}{4}, \frac{f+6 d}{4}\right)$. We show next that $\gamma^{*}$ is the secessionproof allocation that lexicographically maximizes the distribution of costsavings. We also have the following:

$$
e\left(\gamma^{*}, S\right)= \begin{cases}\frac{f}{2} & \text { if } S \in\{\{13\},\{24\},\{23\},\{14\}\} \\ \frac{-2 d}{4} & \text { if } S \in\{\{123\},\{234\},\{134\},\{124\}\} \\ \frac{f f^{2}-6 d}{4} & \text { if } S \in\{\{12\},\{34\}\} \\ \frac{3 f-2 d}{4} & \text { if } S \in\{\{1\},\{4\}\} \\ \text { if } S \in\{\{2\},\{3\}\}\end{cases}
$$

It is not difficult to show that

$$
\min _{S \subset N}\left\{e\left(\gamma^{*}, S\right)\right\}=\frac{f-2 d}{4} .
$$

Thus, note that, because $f \geq 2 d, e\left(\gamma^{*}, S\right) \geq 0$ for all $S \subset N$. As $e\left(\gamma^{*}, N\right)=0$, it follows that $\gamma^{*}$ is secession-proof. Let $\gamma=\left(\gamma_{1}, \gamma_{2}, \gamma_{3}, \gamma_{4}\right)$ be the secession-proof allocation that lexicographically maximizes the distribution of cost-savings. Then, $e(\gamma, S) \geq \frac{f-2 d}{4}$ for all $S \subset N$. Assume, by contradiction, that there is a three-agent coalition $S_{0}$ for which $e\left(\gamma, S_{0}\right)>\frac{f-2 d}{4}$. Then, we would have the following:

$$
\begin{aligned}
& \gamma_{1}+\gamma_{2}+\gamma_{3} \leq \frac{3 f+10 d}{4}=e(\gamma,\{123\})-\frac{f-2 d}{4} \\
& \gamma_{2}+\gamma_{3}+\gamma_{4} \leq \frac{3 f+10 d}{4} \\
& \gamma_{1}+\gamma_{3}+\gamma_{4} \leq \frac{3 f+14 d}{4} \\
& \gamma_{1}+\gamma_{2}+\gamma_{4} \leq \frac{3 f+14 d}{4}
\end{aligned}
$$

with at least one strict inequality. Thus, $\gamma_{1}+\gamma_{2}+\gamma_{3}+\gamma_{4}<f+4 d$. which would contradict secession-proofness. Consequently, $e(\gamma, S)=\frac{f-2 d}{4}$ for all three-agent coalition $S$. By this, it follows that

$$
\gamma_{1}+2 \gamma_{2}=\frac{3 f+10 d}{4} \text { and } 2 \gamma_{1}+\gamma_{2}=\frac{3 f+14 d}{4}
$$


from where we obtain that $\gamma_{1}=\frac{f+6 d}{4}$, and $\gamma_{2}=\frac{f+2 d}{4}$, which proves that $\gamma=\gamma^{*}$.

\section{The five-agent case}

Let now $N=\{1,2,3,4,5\}$. We know from Proposition 3 that $N$-Efficiency holds in this setting if and only if $f \geq 3 d$. Then, we have the following:

Lemma 4: The nucleolus is given by

$$
\begin{gathered}
\left(\frac{f+7 d}{4}, \frac{f+3 d}{4}, d, \frac{f+3 d}{4}, \frac{f+7 d}{4}\right) \text { if } 3 d \leq f \leq 5 d \\
\left(\frac{f}{5}, \frac{f}{5}, \frac{f}{5}, \frac{f}{5}, \frac{f}{5}\right)+(2 d, d, 0, d, 2 d) \text { if } f>5 d
\end{gathered}
$$

In order to prove this lemma, assume first that $3 d \leq f \leq 5 d$ and let $\gamma^{*}=\left(\frac{f+7 d}{4}, \frac{f+3 d}{4}, d, \frac{f+3 d}{4}, \frac{f+7 d}{4}\right)$. We have the following:

$$
e\left(\gamma^{*}, S\right)= \begin{cases}\frac{3 f-7 d}{4} & \text { if } S=\{1\} \\ \frac{3(f-d)}{4} & \text { if } S \in\{\{2\},\{13\},\{23\}\} \\ \frac{f-3}{2} & \text { if } S=\{3\} \\ \frac{f-d}{2} & \text { if } S \in\{\{12\},\{123\}\} \\ \frac{f+d}{2} & \text { if } S \in\{\{14\},\{134\},\{234\},\{135\}\} \\ \frac{f-d}{4} & \text { if } S \in\{15\},\{24\}\} \\ d & \text { if } S=\{1245\},\{124\},\{1234\},\{1345\}\}\end{cases}
$$

By symmetry, we can obtain $e\left(\gamma^{*}, S\right)$ for the remaining coalitions that do not appear above. Because $f \leq 5 d$, it is straightforward to show that

$$
\min _{S}\left\{e\left(\gamma^{*}, S\right)\right\}=\frac{f-3 d}{2}
$$

Thus, as $f \geq 3 d, e\left(\gamma^{*}, S\right) \geq 0$ for all $S \subset N$ and $e\left(\gamma^{*}, N\right)=0$, which shows that $\gamma^{*}$ is secession-proof. Let $\gamma=\left(\gamma_{1}, \gamma_{2}, \gamma_{3}, \gamma_{4}, \gamma_{5}\right)$ be the secession-proof allocation that lexicographically maximizes the distribution of cost-savings. Then, $e(\gamma, S) \geq \frac{f-3 d}{2}$ for all $S \subset N$. In particular,

$$
e(\gamma,\{123\}) \geq \frac{f-3 d}{2} \text { and } e(\gamma,\{45\}) \geq \frac{f-3 d}{2} .
$$


Assume, by contradiction, that one of the two above inequalities is strict. Then, we would have the following:

$$
\gamma_{1}+\gamma_{2}+\gamma_{3} \leq \frac{f+7 d}{2} \text { and } \gamma_{4}+\gamma_{5} \leq \frac{f+5 d}{2}
$$

with at least one strict inequality. Thus, $\sum_{i \in N} \gamma_{i}<f+6 d$, which would contradict secession-proofness. Consequently, $e(\gamma,\{123\})=e(\gamma,\{45\})=\frac{f-3 d}{2}$. By a symmetric argument, we can show that $e(\gamma,\{12\})=e(\gamma,\{345\})=\frac{f-3 d}{2}$. Thus, it follows that

$$
\gamma_{1}+\gamma_{2}=\frac{f+5 d}{2} \text { and } \gamma_{1}+\gamma_{2}+\gamma_{3}=\frac{f+7 d}{2},
$$

from where we obtain that

$$
\gamma_{3}=d
$$

Now, let $\mathcal{S}$ be the set of coalitions whose cost-savings is minimal under $\gamma^{*}$, i.e.,

$$
\mathcal{S}=\left\{S: e\left(\gamma^{*}, S\right)=\frac{f-3 d}{2}\right\}=\{\{123\},\{345\},\{12\},\{45\}\} .
$$

Then, it is not difficult to show that

$$
\min _{S \notin \mathcal{S}}\left\{e\left(\gamma^{*}, S\right)\right\}=\frac{f-d}{4}
$$

Thus, because $\gamma$ is the cost-allocation that lexicographically maximizes the distribution of cost-savings, we have that $e(\gamma, S) \geq \frac{f-d}{4}$ for all $S \notin \mathcal{S}$. Let $\widehat{\mathcal{S}}=\{\{1345\},\{1234\},\{1235\},\{2345\}\}$. Assume, by contradiction, that $e\left(\gamma^{*}, S\right)>\frac{f-d}{4}$ for some $S \in \widehat{\mathcal{S}}$. Then, we would have the following:

$$
\begin{aligned}
& \gamma_{1}+\gamma_{3}+\gamma_{4}+\gamma_{5} \leq \frac{3(f+7 d)}{4} \\
& \gamma_{1}+\gamma_{2}+\gamma_{3}+\gamma_{4} \leq \frac{3 f+17 d}{4} \\
& \gamma_{1}+\gamma_{2}+\gamma_{3}+\gamma_{5} \leq \frac{3(f+7 d)}{4} \\
& \gamma_{2}+\gamma_{3}+\gamma_{4}+\gamma_{5} \leq \frac{3 f+17 d}{4}
\end{aligned}
$$

with at least one strict inequality. Thus, by (25),

$$
3\left(\gamma_{1}+\gamma_{2}+\gamma_{4}+\gamma_{5}\right)<3 f+15 d
$$

from where it follows that

$$
\sum_{i \in N} \gamma_{i}=\left(\gamma_{1}+\gamma_{2}+\gamma_{4}+\gamma_{5}\right)+d<f+6 d,
$$


which contradicts secession-proofness. Consequently, $e(\gamma, S)=\frac{f-d}{4}$ for all $S \in \widehat{\mathcal{S}}$. Then, it follows that

$$
\begin{aligned}
& \gamma_{1}+\gamma_{4}+\gamma_{5}=\gamma_{1}+\gamma_{2}+\gamma_{5}=\frac{3 f+17 d}{4} \\
& \gamma_{1}+\gamma_{2}+\gamma_{4}=\gamma_{2}+\gamma_{4}+\gamma_{5}=\frac{3 f+13 d}{4}
\end{aligned}
$$

Equivalently,

$$
2 \gamma_{1}+\gamma_{2}=\frac{3 f+17 d}{4}, \gamma_{1}+2 \gamma_{2}=\frac{3 f+13 d}{4}, \gamma_{1}=\gamma_{5}, \gamma_{2}=\gamma_{4}
$$

Hence, $\gamma_{1}=\frac{f+3 d}{4}=\gamma_{5}$, and $\gamma_{2}=\frac{f+7 d}{4}=\gamma_{4}$, which proves that $\gamma=\gamma^{*}$

Assume now that $f>5 d$ and let $\gamma^{*}=\left(\frac{f}{5}, \frac{f}{5}, \frac{f}{5}, \frac{f}{5}, \frac{f}{5}\right)+(2 d, d, 0, d, 2 d)$. We have the following:

$$
e\left(\gamma^{*}, S\right)= \begin{cases}\frac{4 f}{5}-2 d & \text { if } S=\{1\} \\ \frac{4 f}{5}-d & \text { if } S=\{2\} \\ \frac{4 f}{5} & \text { if } S=\{3\} \\ \frac{3 f}{5}-2 d & \text { if } S=\{12\} \\ \frac{3 f}{5} & \text { if } S \in\{\{23\},\{13\},\{14\},\{15\},\{24\}\} \\ \frac{2 f}{5}-d & \text { if } S \in\{\{123\},\{124\},\{125\}\} \\ \frac{2 f}{5} & \text { if } S \in\{\{234\},\{134\},\{135\}\} \\ \frac{f}{5} & \text { if } S=N \backslash\{k\}\end{cases}
$$

Note that, by symmetry, we can obtain $e\left(\gamma^{*}, S\right)$ for the remaining coalitions that do not appear above. Because $f>5 d$, it is straightforward to show that

$$
\min _{S \subset N}\left\{e\left(\gamma^{*}, S\right)\right\}=\frac{f}{5}
$$

Let $\gamma=\left(\gamma_{1}, \gamma_{2}, \gamma_{3}, \gamma_{4}, \gamma_{5}\right)$ be the secession-proof allocation that lexicographically maximizes the distribution of cost-savings. Then, $e(\gamma, S) \geq \frac{f}{5}$ for all $S \subset N$. In particular, $e(\gamma, N \backslash\{k\}) \geq \frac{f}{5}$ for all $k$. Assume, by contradiction, that $e(\gamma, N \backslash\{k\})>\frac{f}{5}$ for some $k$. Then, we would have the following:

$$
\begin{aligned}
& \gamma_{2}+\gamma_{3}+\gamma_{4}+\gamma_{5} \leq f+4 d-\frac{f}{5} \\
& \gamma_{1}+\gamma_{3}+\gamma_{4}+\gamma_{5} \leq f+5 d-\frac{f}{5} \\
& \gamma_{1}+\gamma_{2}+\gamma_{4}+\gamma_{5} \leq f+6 d-\frac{f}{5} \\
& \gamma_{1}+\gamma_{2}+\gamma_{3}+\gamma_{4} \leq f+5 d-\frac{f}{5} \\
& \gamma_{1}+\gamma_{2}+\gamma_{3}+\gamma_{4} \leq f+4 d-\frac{f}{5}
\end{aligned}
$$


with at least one strict inequality. Thus, $4\left(\gamma_{1}+\gamma_{2}+\gamma_{3}+\gamma_{4}+\gamma_{5}\right)<4 f+24 d$, from where it follows that $\sum_{i \in N} \gamma_{i}<f+6 d$, which contradicts secession proofness. Consequently, $e(\gamma, S)=\frac{f}{5}$ for all $S \in \widehat{\mathcal{S}}$. Then, it follows that

$$
\begin{aligned}
& f+6 d-\gamma_{1}=f+6 d-\gamma_{5}=\frac{4 f}{5}+4 d \\
& f+6 d-\gamma_{2}=f+6 d-\gamma_{4}=\frac{4 f}{5}+5 d \\
& f+6 d-\gamma_{3}=\frac{4 f}{5}+6 d
\end{aligned}
$$

Hence, $\gamma_{1}=\frac{f+10 d}{5}=\gamma_{5}, \gamma_{2}=\frac{f+5 d}{5}=\gamma_{4}$, and $\gamma_{3}=\frac{f}{5}$, which proves that $\gamma=\gamma^{*}$

\section{The six-agent case}

Let now $N=\{1,2,3,4,5,6\}$. We know from Proposition 3 that $N$-Efficiency holds in this setting if and only if $f \geq 5 d$. Then, we have the following:

Lemma 5: The nucleolus is given by

$$
\left(\frac{f}{6}, \frac{f}{6}, \frac{f}{6}, \frac{f}{6}, \frac{f}{6}, \frac{f}{6}\right)+\left(\frac{5}{2} d, \frac{3}{2} d, \frac{1}{2} d, \frac{1}{2} d, \frac{3}{2} d, \frac{5}{2} d\right)
$$

We have the following:

$$
e\left(\gamma^{*}, S\right)= \begin{cases}\frac{5}{6}(f-3 d) & \text { if } S \in\{\{1\},\{6\}\} \\ \frac{5}{6}\left(f-\frac{9}{5} d\right) & \text { if } S \in\{\{2\},\{5\}\} \\ \frac{5}{6}\left(f-\frac{3}{5} d\right) & \text { if } S \in\{\{3\},\{4\}\} \\ \frac{2}{3} f-3 d & \text { if } S \in\{\{12\},\{56\}\} \\ \frac{2}{3} f-d & \text { if } S \in\{\{13\},\{46\}\} \\ \frac{2}{3} f & \text { if } S \in\{\{14\},\{36\},\{15\},\{26\},\{16\},\{23\},\{45\},\{24\},\{35\},\{25\},\{34\}\} \\ \frac{1}{2}(f-5 d) & \text { if } S \in\{\{123\},\{456\}\} \\ \frac{1}{2}(f-3 d) & \text { if } S \in\{\{124\},\{356\},\{125\},\{256\},\{126\},\{156\}\} \\ \frac{1}{2}(f-d) & \text { if } S \in \mathcal{S}_{1} \\ \frac{1}{3}(f-3 d) & \text { if } S \in\{\{1234\},\{3456\},\{1235\},\{2456\},\{1236\},\{1456\}\} \\ \frac{f}{3} & \text { if } S \in \mathcal{S}_{2} \\ \frac{1}{6}(f-3 d) & \text { if }|S|=5\end{cases}
$$

where

$\mathcal{S}_{1}=\{\{134\},\{346\},\{135\},\{246\},\{136\},\{146\},\{145\},\{236\},\{234\},\{345\},\{235\},\{245\}\}$, 
and

$\mathcal{S}_{2}=\{\{1245\},\{2356\},\{1246\},\{1356\},\{1256\},\{1345\},\{2346\},\{1346\},\{2345\}\}$.

It is not difficult to show that

$$
\min _{S \subset N}\left\{e\left(\gamma^{*}, S\right)\right\}= \begin{cases}\frac{1}{2}(f-5 d) & \text { if } 5 d \leq f \leq 6 d \\ \frac{1}{6}(f-3 d) & \text { if } f>6 d\end{cases}
$$

Thus, we distinguish two cases.

- Case 1: $5 d \leq f \leq 6 d$

Let $\gamma=\left(\gamma_{1}, \gamma_{2}, \gamma_{3}, \gamma_{4}, \gamma_{5}, \gamma_{6}\right)$ be the secession-proof allocation that lexicographically maximizes the distribution of cost-savings. Then, $e(\gamma, S) \geq$ $\frac{1}{2}(f-5 d)$ for all $S \subset N$. Assume that either $e(\gamma,\{123\})>\frac{1}{2}(f-5 d)$, or $e(\gamma,\{456\})>\frac{1}{2}(f-5 d)$. Then, we would have the following:

$$
\gamma_{1}+\gamma_{2}+\gamma_{3} \leq \frac{f+9 d}{2} \text { and } \gamma_{6}+\gamma_{5}+\gamma_{4} \leq \frac{f+9 d}{2}
$$

with at least one strict inequality. Thus, $\sum_{i \in N} \gamma_{i}<f+9 d$, which contradicts secession-proofness. Consequently, $e(\gamma,\{123\})=\frac{1}{2}(f-5 d)=e(\gamma,\{456\})$.

Given the distribution of cost-savings associated to $\gamma^{*}$ provided above, we know that $e(\gamma, S) \geq \frac{1}{6}(f-3 d)$ for all $S \notin\{\{123\},\{456\}\}$. Assume that there exists a five-agent coalition $S$ for which $e(\gamma, S)>\frac{1}{6}(f-3 d)$. Then, we would have the following:

$$
\begin{aligned}
& \gamma_{2}+\gamma_{3}+\gamma_{4}+\gamma_{5}+\gamma_{6} \leq \frac{5 f}{6}+\frac{13 d}{2} \\
& \gamma_{1}+\gamma_{3}+\gamma_{4}+\gamma_{5}+\gamma_{6} \leq \frac{5 f}{6}+\frac{15 d}{2} \\
& \gamma_{1}+\gamma_{2}+\gamma_{4}+\gamma_{5}+\gamma_{6} \leq \frac{5 f}{6}+\frac{17 d}{2} \\
& \gamma_{1}+\gamma_{2}+\gamma_{3}+\gamma_{5}+\gamma_{6} \leq \frac{5 f}{6}+\frac{17 d}{2} \\
& \gamma_{1}+\gamma_{2}+\gamma_{3}+\gamma_{4}+\gamma_{6} \leq \frac{5 f}{6}+\frac{15 d}{2} \\
& \gamma_{1}+\gamma_{2}+\gamma_{3}+\gamma_{4}+\gamma_{5} \leq \frac{5 f}{6}+\frac{13 d}{2}
\end{aligned}
$$

with at least one strict inequality. Thus, $5 \sum_{i \in N} \gamma_{i}<5 f+45 d$, which contradicts secession-proofness. Consequently, $e(\gamma, S)=\frac{1}{6}(f-3 d)$ for all five-agent coalition $S$. Thus,

$$
\begin{aligned}
& f+9 d-\gamma_{1}=f+9 d-\gamma_{6}=\frac{5 f}{6}+\frac{13 d}{2} \\
& f+9 d-\gamma_{2}=f+9 d-\gamma_{5}=\frac{5 f}{6}+\frac{15 d}{2} \\
& f+9 d-\gamma_{3}=f+9 d-\gamma_{4}=\frac{5 f}{6}+\frac{17 d}{2}
\end{aligned}
$$

from where we easily obtain that $\gamma_{k}=\gamma_{k}^{*}$ for all $k$. 
- Case 2: $f>6 d$

Let $\gamma=\left(\gamma_{1}, \gamma_{2}, \gamma_{3}, \gamma_{4}, \gamma_{5}, \gamma_{6}\right)$ be the secession-proof allocation that lexicographically maximizes the distribution of cost-savings. Then, $e(\gamma, S) \geq$ $\frac{1}{6}(f-3 d)$ for all $S \subset N$. Assume, by contradiction, that there exists a five-agent coalition $S$ such that $e(\gamma, S)>\frac{1}{6}(f-3 d)$. Then, an analogous argument to the one made in Case 1 concludes.

\section{References}

Alesina, A., Angeloni, I. and F. Etro (2005), "International unions", American Economic Review 95, 602-615.

Arin, J. and E. Iñarra (2001), "Egalitarian solutions in the core", International Journal of Game Theory 30, 187-193.

Atkinson, A.B. (1970), "On the measurement of inequality", Journal of Economic Theory 2, 244-263.

Aumann, R.J. and M. Maschler (1985), "Game Theoretic Analysis of a Bankruptcy Problem from the Talmud", Journal of Economic Theory 36, 195-213.

Balinski, M.L. (1965), "Integer programming: methods, uses, computation", Management Science 12, 253-313.

Bichchandani, S. and J.W. Mamer (1997), "Equilibrium in an exchange economy with indivisiblities", Journal of Economic Theory 74, 385-413.

Bichchandani, S. and J.M. Ostroy (2002), "The package assignment model", Journal of Economic Theory 107, 377-406.

Blaser, M. and L. Shankar Ram (2008), "Approximately fair cost allocation in metric traveling salesman games", Theory of Computing Systems 43, 19-37.

Bondareva, O. (1962), "Some applications of linera programming methods to the theory of cooperative games" (in Russian0, Problemy Kibernitiky, 10, 119-139.

Chudak, F.A. (1998), "Improved approximation algorithms for uncapacited facility location", in Integer Programming and Combinatorial Optimization, R.E. Bixby, E.A. Boyd and R.Z. Rios-Mercado, eds., Notes in Computer Science 1412, Springer, 180-194.

Chudak, F.A. and D.B. Shmoys (1999), "Improved approximation algorithms for the uncapacited facility location problem", mimeo, Cornell University. 
Clarke, E. H., (1971) Multipart pricing of public goods. Public Choice 11, $17 Đ 33$ (1971).

Cornuéjols, G. Nemhauser, G.L. and L.A. Wolsey (1990), The uncapacited facility location problem" in Discrete Location Theory, P. Mirchandani and R. Francis, eds., John Wiley and Sons, NYC, New York, 119-171.

Dasgupta, P., Sen, A.K. and D.A. Starrett (1973), "Notes on the measurement of inequality", Journal of Economic Theory 6, 180-187.

Devanur, N., M. Mihail, M. and V. Vazirani (2005), "Strategy-proof costsharing mechanisms for set cover and facility location problems", Decision Support Systems 39, 11-22.

Drèze, J., Le Breton, M., Savvateev, A. and S. Weber (2008), "Almost subsidy-free spatial pricing in a multidimensional setting", Journal of Economic Theory 143, 275-291.

Driessen, T.S.H. and S.H. Tijs (1986), "Extensions of solution concepts by means of multiplicative $\varepsilon$-tax games", Mathematical Social Sciences 12 , 9-20.

Dutta, B. and D. Ray (1989), "A concept of egalitarianism under participation constraints" Econometrica 57, 615-635.

Faigle, U. and W. Kern (1993), "On some approximately balanced combinatorial cooperative games", ZOR Mathematical Methods of Operations Research 38, 141-152

Faigle, U. and W. Kern (1998), "Approximate core allocations for bin packing games", Siam Journal of Discrete Mathematics 11, 397-399.

Faigle, U., Fekete, S.P., Kern, W. and W. Hochstattler (1998), "The nucleon of cooperative games and an algorithm for matching games", Mathematical Programming 83, 195-211.

Faigle, U., Kern, W. and D. Paulusma (2000), "Note on the computational complexity of least core concepts for min-cost spanning tree games", Mathematics Methods of Operations Research 52, 23-38

Goemans, M.X. and M Skutella (2004), "Cooperative facility location games", Journal of Algorithms 50, 192-214.

Granot, D. and G. Huberman (1981), "On minimum cost spanning tree games", Mathematical Programming 21, 1-18.

Granot, D. and G. Huberman (1984), "On the core and nucleolus of minimum cost spanning tree games", Mathematical Programming 29, 323347.

Green, J., Kohlberg, E., Laffont, J.J., (1976), "Partial equilibrium approach to the free rider problem", Journal of Public Economics 6, 375 Đ394

Groves, T., (1973), "Incentives in teams". Econometrica 41, 617-663. 
Grotte, J.H. (1970), "Computation of and observations on the nucleolus, the normalized nucleolus and the central games",M. Sc. Thesis, Cornell University, Ithaca, NY.

Guha, S. and S. Khuller (1998), "Greedy strikes back: improved facility location algorithms". in Proceedings of the 9th Annual ACM-SIAM Symposium on Discrete Algorithms, 649-657.

Hajiaghayi, M.T., Madhian, M. and V.S. Mirrokni (2003), "The facility location problem with general cost functions", Networks 42, 42-47.

Hougaard, J.L., Thorlund-Petersen, L. and B. Peleg (2001), "On the set of Lorenz-maximal imputations in the core of a balanced game", International Journal of Game Theory 30, 147-165.

Immorlica, N., Mahdian, M and V. Mirrokni (2008), "Limitations of cross-monotonic cost-sharing schemes", ACM Transactions on Algorithms, $1-25$.

Jain, K., Mahdian, M., Markakis, E., Saberi, A. and V.V. Vazirani (2003), "Greedy facility location algorithms analyzed using dual fitting with factor revealing LP", Journal of the ACM 50, 795-824.

Jain, K. and V.V. Vazirani (2001), "Approximation algorithms for metric facility location and k-median problems using the primal-dual schema and Lagrangian relaxation", Journal of the ACM 48, 274-296.

Kelso, A. and V.P. Crawford (1982), "Job-Matching, Coalition Formation and Gross Substitutes", Econometrica 50, 1483-1504.

Kern, W. and D. Paulusma (2009), "On the core and f-nucleolus of flow games", Mathematics of Operations Research, forthcoming.

Kuipers, J. (1998), "Bin packing games", Mathematical Methods of Operations Research 47, 499-510.

Kolen, A. (1983), "Solving covering problems and the uncapacited plant location problem on trees", European Journal of Operational Research 12, 266-278.

Le Breton, M. and S. Weber (2003) "The art of making everybody happy: how to prevent a secession?", IMF Staff Papers 50, 403-435.

Littlechild, S. (1974), "A simple expression for the nucleolus in a special case", International Journal of Game Theory 3, 21-30.

Madhian, M., Ye, Y. and J. Zhang (2002), "Improved approximation algorithms for metric facility location problems", in The 5th International Workshop on Approximation Algorithms for Combinatorial Optimization, 229-242.

Makowski, L. and J.M. Ostroy (2000), "Linear programming and general equilibrium theory", UCLA, mimeo. 
Maschler, M., Peleg, B. and L.S. Shapley (1979), "Geometric properties of the kernel, nucleolus and related solution concepts", Mathematics of Operations Research 4, 303-338.

Mas-Colell, A. (1980), "Efficiency and decentralization in the pure theory of public goods", Quarterly Journal of Economics 94, 625-641.

Mirchandani, P. and R. Francis (19990), Discrete Location Theory, John Wiley and Sons, NYC, New York.

Montero, M. (2006), "Noncooperative foundations of the nucleolus in majority games", Games and Economic Behavior 54, 380-397.

Moulin, H. (1999), "Incremental cost sharing: characterization by coalition strategy-proofness", Social Choice and Welfare 16, 279-320.

Moulin, H. (2009), "Almost budget-balanced VCG mechanisms to assign multiple objects", Journal of Economic Theory 144, 96-119.

Moulin, H., Shenker, S., (2001) "Strategy-proof sharing of submodular costs: Budget balance versus efficiency", Economic Theory 18, 511-533.

Owen, G. (1974), "A Note on the nucleolus", International Journal of Game Theory 3, 101-103.

Owen, G. (1975), "The Core of linear production games", Mathematical Programming 9, 358-370.

Owen, G. (2001) Game Theory, Third Edition, Academic Press, NYC, New York.

Pal, M. and E. Tardos (2003), "Group strategy-proof mechanisms via primal-dual algorithms", in the Proceedings of the Annual IEEE Symposium on Foundations of Computer Science, 584-593.

Peleg, B. (1968), "On weights of constant-sum majority games", SIAM Journal on Applied Mathematics 16, 527-532.

Peleg, B. and P. Sudhölter (2003), Introduction to the Theory of Cooperative Games, Kluwer Academic Publishers, Boston, MA.

ReVelle, C.S., Eiselt, H.A. and M.S. Daskin (2008), "A bibliography for some fundamental problem categories in discrete location science", European Journal of Operational Research 134, 817-848..

Rotschild, M. and J. Stiglitz (1973), "Some further results on the measurement of inequality", Journal of Economic Theory 6, 188-204.

Samet, D. and E. Zemel (1984), "On the core and dual set of linear programming games", Mathematics of Operations Research 9, 309-316.

Schmeidler, D. (1969), "The nucleolus of a characteristic function game", Siam Journal of Applied Mathematics 17, 1163-1170.

Shapley, L.S. (1953) "A value for n-person games", in Contributions to the Theory of Games, Kuhn H.W. and A.W. Tucker, eds., volume II, 307317 . 
Shapley, L.S. (1967), "On balanced sets and cores", Naval Research Logistics Quarterly 14, 453-460.

Shapley, L.S. and M. Shubik (1971), "Assignment games I: the core" International Journal of Game Theory 1, 111-130.

Shmoys, D.B., Tardos, E. and K. Aardal (1997), "Approximation algorithms for facility location problems", in Proceedings of the 29th Symposium on Theory of Computing, 265-274.

Sviridenko, M. (2002), "The 1.582-approximation algorithm for the metric uncapacited facility location problem", in The 9th Conference on Integer Programming and Combinatorial Optimization, 240-257.

Tamir, A. (1992), "On the core of cost allocation games defined on location problems", Transportation Science 27, 81-86.

Trubin, V.A. (1976), "An effective algorithm for solving the distribution problem in a network in the form of a tree", Soviet Mathematics Doklady 17, 1604-1608.

Wallmeier, E. (1983), "Der f-Nucleolus und ein dynamisches Verhandlungsmodell als Lösungskonzepte für kooperative N-personenspiele" (in German), Dissertation reprint, Skripten zur Mathematischen Statistik 5, Westfälische Wilhelms-Universität, Münster.

Woeginger, G.J. (1995), "On the rate of taxation in a cooperative bin packing game", ZOR Mathematical Methods of Operations Research 42, 313324 . 